\title{
Probabilistic Data Structures in Adversarial Environments
}

\author{
David Clayton \\ University of Florida \\ Gainesville, Florida \\ davidclayton@ufl.edu
}

\author{
Christopher Patton \\ University of Florida \\ Gainesville, Florida \\ cjpatton@ufl.edu
}

\author{
Thomas Shrimpton \\ University of Florida \\ Gainesville, Florida \\ teshrim@ufl.edu
}

\begin{abstract}
Probabilistic data structures use space-efficient representations of data in order to (approximately) respond to queries about the data. Traditionally, these structures are accompanied by probabilistic bounds on query-response errors. These bounds implicitly assume benign attack models, in which the data and the queries are inputs are chosen non-adaptively, and independent of the randomness used to construct the representation. Yet probabilistic data structures are increasingly used in settings where these assumptions may be violated.

This work provides a provable security treatment of probabilistic data structures in adversarial environments. We give a syntax that captures a wide variety of in-use structures, and our security notions support development of error bounds in the presence of powerful attacks.

Concretely, we primarily focus on examining the widely used Bloom filter, but also consider counting (Bloom) filters and countmin sketch data structures. For the traditional version of these, our security findings are largely negative; however, we show that simple embellishments (e.g., using salts, or secret keys) yields structures that provide provable security, and with little overhead.
\end{abstract}

\section{CCS CONCEPTS}

- Security and privacy $\rightarrow$ Symmetric cryptography and hash functions.

\section{KEYWORDS}

Data structures, Bloom filters, Count min-sketches, counting Bloom filters

\section{ACM Reference Format:}

David Clayton, Christopher Patton, and Thomas Shrimpton. 2019. Probabilistic Data Structures in Adversarial Environments. In 2019 ACM SIGSAC Conference on Computer and Communications Security (CCS '19), November 11-15, 2019, London, United Kingdom. ACM, New York, NY, USA, 18 pages. https://doi.org/10.1145/3319535.3354235

\section{INTRODUCTION}

Probabilistic data structures, which use space-efficient representations of data to provide (approximately correct) answers to queries about the data, find myriad uses in modern communication, storage,

Permission to make digital or hard copies of all or part of this work for personal or classroom use is granted without fee provided that copies are not made or distributed for profit or commercial advantage and that copies bear this notice and the full citation on the first page. Copyrights for components of this work owned by others than ACM must be honored. Abstracting with credit is permitted. To copy otherwise, or republish, to post on servers or to redistribute to lists, requires prior specific permission and/or a fee. Request permissions from permissions@acm.org.

CCS '19, November 11-15, 2019, London, United Kingdom

(c) 2019 Association for Computing Machinery.

ACM ISBN 978-1-4503-6747-9/19/11 . \$15.00

https://doi.org/10.1145/3319535.3354235 and computational systems. The Bloom filter [4], for example, is ubiquitous in distributed computing, including web caches (e.g., Squid) and hash tables (e.g., BigTable and Hadoop), resource and packet routing, and network measurement. (We refer the reader to the surveys $[5,28]$ for a comprehensive list of applications.)

The traditional approach to analyzing the correctness of a data structure is to assume that all inputs, and all queries, are independent of any internal randomness used to construct it. But as highlighted by Naor and Yogev (CRYPTO '15 [24]), there are important use-cases in which the inputs and queries may be chosen adversarially and adaptively, based on partial information and prior observations about the data structure. Attacks of this sort can be used to disrupt or reduce the availability of real systems [9, 18, 22].

Naor and Yogev (hereafter NY) formalized a notion of adversarial correctness for Bloom-filter-like structures. Recall that a Bloom filter encodes a set $\mathcal{S}$ into a length- $m$ array of bits (initially all zeros), where $m$ is much less than the number of bits needed to store $\mathcal{S}$ in full. Elements $x \in \mathcal{S}$ are encoded by computing multiple hash values $h_{1}(x), h_{2}(x), \ldots, h_{k}(x) \in[m]$, then setting the indicated array positions to 1 . This bit-array representation of $\mathcal{S}$ allows for set membership queries, i.e., "is $x \in \mathcal{S}$ ?", by hashing $x$ and responding positively iff all of the indicated positions hold a 1-bit. False-negative respones are not possible, but false-positive responses are. Classical results relate $|\mathcal{S}|, m, k$ to the probability of false-positive query responses $[5,20]$, where the probability is over the sampling of the hash functions. (These are usually modeled as independent random functions.) Crucially, these results assume that $\mathcal{S}$ and the $h_{1}, \ldots, h_{k}$ are independent of each other. Said another way, even if $\mathcal{S}$ is adversarially chosen, this choice cannot depend on particular hash functions that are used to produce the Bloom filter and compute the query responses. The conceptual innovation of NY was remove this assumption an explore the consequences upon the probability of Bloom filter query-response errors. In particular, NY allowed the adversary to specify a (fixed) set $\mathcal{S}$ that may depend on the hash functions, and then attempt to induce errors via set-membership queries.

We expand upon NY in several ways, providing syntax and security notions that allow analysis of a large class of data structures (not only Bloom filters), in settings where the data may not be a set and may change over time, and where the structure's representation of the data may (or may not) be publicly visible.

BEYOND SETS AND BLOOM FILTERS. Our first significant extension of NY is that our attack model allows the adversary to adaptively update the collection $\mathcal{S}$ during its attack. This captures settings in which the data to be represented may change over time, e.g., streaming data applications. Many data structures are designed for such settings - the counting filter [15], count-min sketch [8], cuckoo filter [14], and stable Bloom filter [11], to name a few - 
by providing updatable, or mutable, representations. Our syntactic formalization of data structures captures this reality.

Next, while the Bloom filter was designed to represent data collections $\mathcal{S}$ that are sets, streaming data (for example) is more accurately modeled as a multiset. Here one is often interested in information about frequency, e.g., "how many times does $x$ appear in $\mathcal{S}$ ?" Thus, in addition to admiting mutable respresentation, our formalization of data structures allows for rich query spaces. Specifically, we define a data structure to be a triple of algorithms (REP, QRY, UP) denoting the representation, query-evaluation, and update algorithms, respectively. Associated to the data structure is a set of supported query functions $\mathcal{Q}$, and a set $\mathcal{U}$ of allowed update functions. For reasons we will elucidate in a moment, all three algorithms take a key $K$ as input, and both REP and UP may be randomized.

The combination of mutability and rich query spaces has significant implications for security. Consider the counting filter structure [15]. It is similar to a Bloom filter, but instead of a bit array, a counting filter represents an updatable multiset $\mathcal{S}$ as an array of $m$ integers; these serve as counters. To add $x$ to $\mathcal{S}$, hash values $h_{1}(x), \ldots, h_{k}(x) \in[m]$ are computed, and the indicated counters are incremented. Decrementing the counters implements deletion of an occurrence of $x$ from $\mathcal{S}$. Like a Bloom filter, a counting filter provide approximately correct answers to set-membership queries ${ }^{1}$, where a query about $x$ results in a positive response iff all of the hash-indicated counters are at least one.Unlike a Bloom filter, this structure admits both false-positive and false-negative responses. In particular, if the representation is updated by "removing" an element $y$ that does not appear in the underlying $\mathcal{S}$, one or more of the counters associated to $x$ may be decremented, potentially causing $x$ to become a false negative.

Both the Bloom and counting filters have binary query responses, making the notion of response error easy to define: the response is correct, or it isn't. But practically important structures, like the count-min sketch, admit frequency-of-element queries, which have integer responses. What constitutes an error is less clear, when this is the case. Even in the traditional analyses (i.e., non-adaptive attacks) one is guaranteed only that responses will be "close" to correct, with probability "close" to one. We therefore parameterize our security experiments with a specifiable error function $\delta$. If the correct response to an adversarial query is $a$ and the data structure responds with $a^{\prime}$, the experiments award the adversary with an error weight $\delta\left(a, a^{\prime}\right) \geq 0$. Our experiments are additionally parameterized by an error capacity $r \geq 0$, and the adversary is considered to "win" if the total cost of the errors it induces is greater than this value. As it turns out, even calculating this total cost is not straightforward in our setting: one must determine whether or not the cost of a given error should be carried across (adaptive, adversarial) updates to $\mathcal{S}$ and its representation.

Public vs. Private Representations. We define two experiments, one in which representations are shown to the adversary, and one in which they are not. In the ERR-Pub game, the adversary is given a representation-oracle $\operatorname{Rep}$ that, on input a collection $\mathcal{S}$, returns the resulting representation $\operatorname{ReP}_{K}(\mathcal{S})$. Note that the key $K$ (which

\footnotetext{
${ }^{1}$ Indeed, they were initially introduced to support deletions from a set, without having to rebuild the representation, as one would for a Bloom filter.
}

\begin{tabular}{|l|l|}
\hline Structure & Results \\
\hline \multirow{3}{*}{$\begin{array}{l}\text { Bloom filter } \\
\text { (Fig. 5, Fig. 7) }\end{array}$} & $\begin{array}{l}\text { Immutitional implementation insecure. } \\
\text { with per-representation salt. Mutable case: } \\
\text { structure additionally require a secret key or } \\
\text { keeping representations private, and benefit } \\
\text { from thresholding. }\end{array}$ \\
\hline \multirow{3}{*}{$\begin{array}{l}\text { Counting filter (Fig. 9) } \\
\text { Count-min sketch }\end{array}$} & $\begin{array}{l}\text { Traditional implementation insecure. } \\
\text { representation salt, thresholding, and private } \\
\text { representations. }\end{array}$ \\
\hline Fig) & $\begin{array}{l}\text { Traditional implementation insecure. } \\
\text { Security can be achieved by combining a per- } \\
\text { representation salt, thresholding, and private } \\
\text { representations. }\end{array}$ \\
\hline
\end{tabular}

Figure 1: A high-level, informal summary of our results.

may be the empty string, to capture unkeyed structures) is fixed across all calls; however, per-representation randomness (e.g. salts) may be present. The adversary is permitted to (adaptively) update any established representation via an update-oracle $\mathbf{U p}$ and, at any time, it may query a representation via a query-oracle Qry. The adversary is given credit (determined by $\delta$ ) for each Qry-query that results in an error.

The ERR-Priv game is defined in much the same way, except that representations are not shown to the adversary unless it explicitly asks for them to be revealed.

There are many applications in which the adversary would not have unfettered access to the structure [18], and for which the assumption of a private data structure is most fitting. However, we do not want to rule out the possibility that the adversary may eventually learn the contents of some data structures, which are likely to have looser access controls than long-term private keys or similar critical data. When possible, we would also like to account for cases where the adversary may be able to freely view the data structures as well, such as in the cases where Bloom filters are used for distributed computations. This corresponds to the ERR-Pub case, though this is a much stronger notion which we will find is not always achievable.

CASE STUdies AND OUR FINDINGs. We exercise our syntax and notions by analyzing three important, real-world data structures: Bloom filters [4] (Section 4), count min-sketches [8] (Section 6), and counting filters [15] (Appendix 5), summarized in Figure 2. Our studies examine the basic versions of each, as well as variants that may take a key or a per-representation random salt, and variants that incorporate measures of representation saturation. Each of the basic structures supports different queries and update operations; taken together, they provide interesting coverage of the structure/attack-model landscape.

We find that none of the (basic) structures meets either of our security notions. In particular, if the data being represented, the updates, and the queries all may depend on the choice of hash function, then each of these structures is susceptible to a class of attacks we call target-set coverage attacks (described in Section 4.1). 
These are closely related to pollution attacks against standard Bloom filters [18], which we will discuss in some detail.

On the positive side, we show how these structures can be modified in order to prove security. These modification are conceptually straightforward and intuitive; we do not, however, study the deployment implications of these modifcations.

BLOOM FILTERS, OUR IN-DEPTH STUDY. Due to their wide-spread and varied use (and following NY), we begin with a deep look at Bloom filters. It is well-known that standard Bloom filters do not perform well in adversarial settings [18, 24]; we first corroborate these findings via an explicit ERR-Priv attack (Section 4.1). We then consider the security of several variants of the basic Bloom filter for which we can derive correctness (i.e., security) bounds. The first idea is to generate a short, random salt, which we prepend to the input of the hash. Thus, instead of computing $h_{i}(x)$ for each $1 \leq i \leq k$ we compute $h_{i}(Z \| x)$, where $Z$ is a short (say, 128-bit) string chosen by the representation algorithm. This leads to our first positive result, for this salted Bloom filter, in the public-representation setting when attacks treat representations as immutable (i.e., updates are forbidden); this is Theorem 4.1. Following the traditional approach [5], we model the hash functions as random oracles (ROM) [1]. Our security argument must account for any hash-exploiting precomputation performed by the adversary via the random oracle. This leads to fairly weak bounds, which means that larger filters must be used to achieve a reasonable correctness upper bound (Figure 6) On the other hand, we find far better bounds, even in the mutable setting, if the representation is kept private (Theorem 4.3).

We derive a similarly good bound for keyed Bloom filters, which use a secretly-keyed pseudorandom function (PRF) instead of a hash function (in addition to salts). This result is in the mutable and public-representation setting (Theorem 4.4), the strongest attack model we formalize.

Normally, Bloom filters are considered to be "full" when some pre-determined set size, or capacity, is reached. Indeed, Bloom filter parameters are generally chosen as a function of this maximum capacity [20]. We explore an alternative definition of fullness, whereby the filter is deemed full once the Hamming weight of the filter (i.e., the number of 1s) crosses a pre-determined threshold. While the two definitions are more or less interchangeable in the non-adaptive, traditional setting, we show that this alternative definition has substantial analytical value in adversarial environments. In Theorem 4.5, we reconsider the security of salted BFs in the mutable, private setting, and exhibit substantially tighter bounds. In particular, we find that as long as salts are reasonably large, we can use a 1 kilobyte filter to store 100 objects, while incurring less than one in a million chance of a single false positive. This holds even if the adversary is allowed to completely control the filter's construction and make up to $2^{16}$ queries (see Figure 8).

Count min-sketches. Following the deep dive into Bloom filters in Section 4, we then consider count min-sketches (CMS), which provide a compact representation of a multiset, allowing additions and deletions, and yielding approximate queries for approximate frequency of an element in the multiset. While a count-min sketch hashes in much the same way as a Bloom filter, it uses a 2D array of non-negative integer counters rather than a linear array of bits, allowing the structure to keep track of how many times each counter is incremented. We find that, CMSes are not secure in the public-representation setting, even if we use a salt or use a PRF in place of the hash function. The fact that the adversary can see exactly which filters are incremented or decremented with each update, along with the fact that updates can be trivially reversed (deletion undoes insertion and vice versa) allows the adversary to mount attacks by trial and error even if it lacks the ability to predict in advance where an element will be sent by the hash functions. However, we are able to derive a good correctness in the mutable/private setting (Theorem 6.1), using a per-representation salt and a notion of "fullness" similar to threshold Bloom filters.

Counting FiLters. In Appendix 5, we include a discussion of the security of counting filters. In a loose sense, these fall somewhere between the CMS and Bloom filters: they admit both addition and deletion updates to the representation (like CMS), but only support set-membership queries. Due to their structural similarities, CMS and counting filters exhibit similar security properties (Theorem 5.1).

FUTURE WORK. The focus of this work is the data structures themselves. Even so, we were only able to consider a handful of (important, real-world) examples. We hope that future work will apply our formalisms to the many probabilistic data structures that exist.

Going into a different direction, future work should also address how adversarial correctness impacts high-level protocols that use these probabilistic data structures. A good example is contentdistribution networks [6], where many servers propagate representations of their local cache to their neighbors. (In Section 4 we will touch briefly on the real-world attacks that are possible in this setting.) The Bloom filter family alone has a wide range of practical applications, for example in large database query processing [5], routing algorithms for peer-to-peer networks [26], protocols for establishing linkages between medical-record databases [27], fair routing of TCP packets [16], and Bitcoin wallet synchronization [19]. Analyzing higher-level primitives or protocols will require establishing appropriate syntax and security notions for those, too; hence we leave this for future work. Another interesting direction is to consider what information data structures leak via their public representations. A large variety of data structures with interesting privacy properties have been proposed. For example, variants of Bloom filters that ensure privacy of the query have been studied [3,25]. These prior work leave open the security of more conventional data structures, like those studied in this paper.

\subsection{Related work}

Comparison with NaOr-Yogev. As previously noted, Naor and Yogev [24] were the first to formalize adversarial correctness of Bloom filters. Our work extends theirs significantly in several directions. First, we consider abstract data structures, rather than only set-membership structures. Even with respect to the specific case of correctness for set-membership structures, our work offers several advantages as compared to the Naor-Yogev treatment. One, our syntax distinguishes between the (secret) key and the public portion of a data structure, an important distinction that is missing in their work. Two, the Naor-Yogev definition of correctness allows the adversary to make several queries, some of which may produce incorrect results; the attacker then succeeds if it outputs a fresh 


\begin{tabular}{|l|l|l|l|l|}
\hline Structure & Data Objects & Supported Queries & Supported Updates & Parameters \\
\hline $\begin{array}{l}\text { Bloom } \\
\text { filter (Fig. 5) }\end{array}$ & $\begin{array}{l}\text { Sets } \\
\mathcal{S} \subseteq\{0,1\}^{*}\end{array}$ & $\operatorname{qry}_{x}(\mathcal{S})=[x \in \mathcal{S}]$ & $\operatorname{up}_{x}(\mathcal{S})=\mathcal{S} \cup\{x\}$ & $\begin{array}{l}n, \max |\mathcal{S}| \\
k \text {, \# hash functions } \\
m, \text { array size (bits) }\end{array}$ \\
\hline $\begin{array}{l}\ell \text {-thresholded } \\
\text { Bloom filter } \\
\text { (Fig. 7) }\end{array}$ & $\begin{array}{l}\text { Sets } \\
\mathcal{S} \subseteq\{0,1\}^{*}\end{array}$ & $\operatorname{qry}_{x}(\mathcal{S})=[x \in \mathcal{S}]$ & $\operatorname{up}_{x}(\mathcal{S})=\mathcal{S} \cup\{x\}$ & $\begin{array}{l}\ell, \text { max no. 1s in array } \\
k, \# \text { hash functions } \\
m \text {, array size (bits) }\end{array}$ \\
\hline $\begin{array}{l}\text { Count-min } \\
\text { sketch (Fig. 10) }\end{array}$ & $\begin{array}{l}\text { Multisets } \\
\mathcal{S} \in \text { Func }\left(\{0,1\}^{*}, \mathbb{N}\right)\end{array}$ & $\operatorname{qry}_{x}(\mathcal{S})=\mathcal{S}(x)$ & $\begin{array}{l}\operatorname{up}_{x, 0}(\mathcal{S})(x)=\mathcal{S}(x)+1 \\
\operatorname{up}_{x, 1}(\mathcal{S})(x)=\mathcal{S}(x)-1 \\
\operatorname{up}_{x, b}(\mathcal{S})(y)=\mathcal{S}(y) \text { for } x \neq y\end{array}$ & $\begin{array}{l}\ell, \text { max no. nonzero counters } \\
m, \text { array functions and arrays }\end{array}$ \\
\hline $\begin{array}{l}\text { Counting } \\
\text { filter (Fig. 9) }\end{array}$ & $\begin{array}{l}\text { Multisets } \\
\mathcal{S} \in \operatorname{Func}\left(\{0,1\}^{*}, \mathbb{N}\right)\end{array}$ & $\operatorname{qry}_{x}(\mathcal{S})=[\mathcal{S}(x)>0]$ & $\begin{array}{l}\operatorname{up}_{x, 1}(\mathcal{S})(x)=\mathcal{S}(x)+1 \\
\operatorname{up}_{x,-1}(\mathcal{S})(x)=\mathcal{S}(x)-1 \\
\operatorname{up}_{x, b}(\mathcal{S})(y)=\mathcal{S}(y) \text { for } x \neq y\end{array}$ & $\begin{array}{l}\ell, \text { max no. non-zero counters } \\
k, \text { hash functions } \\
m, \text { array size (counters) }\end{array}$ \\
\hline
\end{tabular}

Figure 2: The data structures that we consider. Each data structure yields a space-efficient representation of its input data object and, in the presence of non-adaptive attacks, provides approximately correct responses to the supported queries. For counting filters and count-min sketches, typical implementations prevent updates that would cause $\mathcal{S}(x)-1<0$.

query that causes an error. This separation seems arbitrary, and we propose instead a parameterized definition in which the attacker succeeds if it can cause a certain number of (distinct) errors during its entire execution. Three, Naor and Yogev analyze the correctness of a new Bloom filter variant of their own design. In contrast, we are mainly interested in analyzing existing, real-world constructions to understand their security.

OTHER RELATED WORKS. There is a long tradition in computer science of designing structures that concisely (but probabilistically) represent data so as to support some set of queries, and each of these structures has its own interesting security characterisitcs [7, 8, 12, 13, 17, 23].

We have already mentioned the ubiquity of Bloom filters in support of efficient network communication and computing protocols. They also find use in security-critical environments, including spam filters, (distributed) denial-of-service attack detection, and deep packet inspection [28]. Recently, Bloom filters were proposed as a means of efficient certificate-revocation list (CRL) distribution [21], a crucial component of public-key infrastructures.

Correctness of data structures in adversarial settings is wellmotivated in the security literature and in practice. Perhaps the earliest published attack on the correctness of a data structure was due to Lipton and Naughton [22] who showed that timing analysis of record insertion in a hash table allows an adversary to adaptively choose elements so as to increase look-up time, effectively degrading a service's performance. Crosby and Wallach [9] exploited hash collisions to increase the average URL load time in Squid, a web proxy used for caching content in order to reduce network bandwidth. More recently, Gerbet et al. [18] described pollution attacks on Bloom filters, whereby an adversary inserts a number of adaptively-chosen elements with the goal of forcing a high falsepositive rate. Although some of their attacks exploit weak (i.e., non-cryptographic) hash functions (as do [9]), their methodology is effective even for good choices of hash functions. They suggest revised parameter choices for Bloom filters (i.e., filter length and number of hashes) in order to cope with their attacks, as well as the use of keyed hash functions. With our more general attack model, however, we will see that a secret key alone does not guarantee correctness.

\section{SYNTAX}

\subsection{Preliminaries}

Let $x \leftarrow \mathcal{X}$ denote sampling $x$ from a set $\mathcal{X}$ according to the distribution associated with $\mathcal{X}$; if $\mathcal{X}$ is finite and the distribution is unspecified, then it is uniform. Let $[i . . j]$ denote the set of integers $\{i, \ldots, j\}$; if $i>j$, then define $[i . . j]=\emptyset$. For all $m$ let $[m]=[1 . . m]$.

Bitstring OPERATIONs. Let $\{0,1\}^{*}$ denote the set of bitstrings and let $\varepsilon$ denote the empty string. Let $X \| Y$ denote the concatenation of bitstrings $X$ and $Y$. For all $m \geq 0$ define $B_{m}$ as the following function. For all $\boldsymbol{x} \in[m]^{*}$ let $B_{m}(\boldsymbol{x})=X_{1} X_{2} \cdots X_{m} \in\{0,1\}^{m}$, where $X_{v}=1$ if and only if $x_{i}=v$ for some $i \in[|x|]$. We call $B_{m}(\boldsymbol{x})$ the bitmap of $\boldsymbol{x}$. Let $X$ and $Y$ be equal-length bitstrings. We write $X \vee Y$ for their bitwise-OR, $X \wedge Y$ for their bitwise-AND, and $X \oplus Y$ for their bitwise-XOR. Let $\neg X=1^{|X|} \oplus X$, and let $w(X)$ denote the Hamming weight of (i.e., the number of $1 \mathrm{~s}$ in) $X$. For an array $\boldsymbol{M}$ of integers, we analogously define $\boldsymbol{w}^{\prime}(\boldsymbol{m})$ to be the number of nonzero integers in the array. We also define zeroes $(m)$ to be a function taking a non-negative integer and returning a length- $m$ integer vector with all entries set to 0 .

Let $\operatorname{Func}(\mathcal{X}, \mathcal{Y})$ denote the set of functions $f: \mathcal{X} \rightarrow \mathcal{Y}$. For every function $f: \mathcal{X} \rightarrow \mathcal{Y}$, define $\mathrm{ID}^{f}:\{\varepsilon\} \times \mathcal{X} \rightarrow \mathcal{Y}$ so that ID $f(\varepsilon, x)=f(x)$ for all $x$ in the domain of $f$. This allows us to talk about using unkeyed hash functions $H$ in places where, syntactically, a function is required to take a key along with its input.

Adversaries. Adversaries are randomized algorithms that expect access to one or more oracles defined by the experiment in which it is executed. We say that an adversary is $t$-time if it halts in $t$ time steps (with respect to some model of computation, which we leave implicit) regardless of its random coins or the responses to its oracle queries. By convention, the adversary's runtime includes the time required to evaluate its oracle queries.

Pseudorandom functions. For sets $\mathcal{X}$ and $\mathcal{Y}$ and a keyspace $\mathcal{K}$, we define a pseudorandom function to be a function $F: \mathcal{K} \times \mathcal{X} \rightarrow \mathcal{Y}$. The intent is for the outputs of the function to appear random for 


\begin{tabular}{|ll|}
\hline$\frac{\operatorname{Exp}_{F}^{\operatorname{prf}}(A)}{b \leftarrow\{0,1\} ; K \leftarrow \mathcal{K}}$ & oracle $\mathbf{F}(x):$ \\
$b^{\prime} \leftarrow A^{\mathbf{F}}$ & if $b=1$ then return $F_{K}(x)$ \\
$\operatorname{return}\left[b=b^{\prime}\right]$ & if $T[x] \neq \perp$ then return $T[x]$ \\
& $T[x] \leftarrow \mathcal{Y}$; return $T[x]$ \\
\hline
\end{tabular}

Figure 3: The PRF experiment used to define the pseudorandomness of function $F$ with key space $\mathcal{K}$.

a fixed choice of key, which is formally captured by the game described in Figure 3. We define the advantage of an adversary $A$ to be $\operatorname{Adv}_{F}^{\operatorname{prf}}(A)=\operatorname{Pr}\left[\operatorname{Exp}_{F}^{\text {prf }}(A)=1\right]$, and the advantage $\mathbf{A d v}_{F}^{\text {prf }}(t, q)$ to be the maximum advantage of any adversary running for $t$ steps and making $q$ queries to the PRF oracle.

\subsection{Data Structures}

Fix non-empty sets $\mathcal{D}, \mathcal{R}, \mathcal{K}$ of data objects, responses and keys, respectively. Let $\mathcal{Q} \subseteq \operatorname{Func}(\mathcal{D}, \mathcal{R})$ be a set of allowed queries, and let $\mathcal{U} \subseteq \operatorname{Func}(\mathcal{D}, \mathcal{D})$ be a set of allowed data-object updates. A data structure is a tuple $\Pi=(\mathrm{REP}, \mathrm{QRY}, \mathrm{UP})$, where:

- $\operatorname{ReP}: \mathcal{K} \times \mathcal{D} \rightarrow\{0,1\}^{*} \cup\{\perp\}$ is a randomized representation algorithm, taking as input a key $K \in \mathcal{K}$ and data object $\mathcal{S} \in \mathcal{D}$, and outputting the representation repr $\in\{0,1\}^{*}$ of $D$, or $\perp$ in the case of a failure. We write this as repr $\longleftarrow \operatorname{REP}_{K}(\mathcal{S})$.

- QRY : $\mathcal{K} \times\{0,1\}^{*} \times \mathcal{Q} \rightarrow \mathcal{R}$ is a deterministic query-evaluation algorithm, taking as input $K \in \mathcal{K}$, repr $\in\{0,1\}^{*}$, and qry $\epsilon$ $\mathcal{Q}$, and outputting an answer $a \in \mathcal{R}$. We write this as $a \leftarrow$ $\mathrm{QRY}_{K}($ repr, qry).

- Up: $\mathcal{K} \times\{0,1\}^{*} \times \mathcal{U} \rightarrow\{0,1\}^{*} \cup\{\perp\}$ is a randomized update algorithm, taking as input $K \in \mathcal{K}$, repr $\in\{0,1\}^{*}$, and up $\in \mathcal{U}$, and outputting an updated representation repr', or $\perp$ in the case of a failure. We write this as repr' $\nleftarrow \mathrm{Up}_{K}($ repr, up).

Allowing each of the algorithms to take a key $K$ lets us separate (in our security notions) any secret randomness used across data structure operations, from per-operation randomness (e.g., salts). Note that our syntax admits the common case of unkeyed data structures, by setting $\mathcal{K}=\{\varepsilon\}$.

We formalize REP as randomized to admit defenses against offline attacks and, as we will see, per-representation randomness will play an important role in achieving our notion of correctness in the presence of adaptive adversaries. Both REP and the UP algorithm can be viewed (informally) as mapping data objects to representations - explicitly so in the case of REP, and implicitly in the case of Up - so we allow Up to make per-call random choices, too. Many common data structures do not have randomized representation updates, but some do, e.g. the Cuckoo filter [14] and the stable Bloom filter [11].

Note that Up takes a function operating on data objects as an argument, even though Up itself operates on representations of data objects. This is intentional, to match the way these data structures generally operate. In a data structure representing a set or multiset, we often think of performing operations such as 'insert $x$ ' or 'delete $y$ '. When the set or multiset is not being stored, but instead modeled via a representation, the representation must transform these operations into operations on the actual data structure it is using for storage. A Bloom filter, for example, will handle an 'insert $x$ ' query by hashing $x$ and setting the resulting bits in the filter to 1 . In this way, the abstract insertion function up ${ }_{x}$, operating on sets, is handled by Up as a concrete action of setting certain bits in the filter. Side-effects of Up, or cases where the algorithm's behavior does not perfectly match the intended update up, are a potential source of errors that an adversary can exploit.

We also note that the query algorithm QRY is deterministic. This reflects the overwhelming behavior of data structures in practice, in particular those with space-efficient representations. It also allows us to focus on correctness errors caused by the actions of an adaptive adversary, without attending to those caused by randomized query responses. Randomized query responses may be of interest from a data privacy perspective, but our focus is on correctness.

\section{NOTIONS OF ADVERSARIAL CORRECTNESS}

Let $\Pi=($ ReP, Up, QRY) be a data structure with response space $\mathcal{R}$. We define two adversarial notions of correctness given by a pair of related experiments for $\Pi$, error function $\delta: \mathcal{R}^{2} \rightarrow \mathbb{R}$, and error capacity $r$. The values $\delta(x, y)$ of the error function represent the "badness" of getting an erroneous result of $x$ from QRY when $y$ should actually have been returned. In general we require $\delta(x, y) \geq$ 0 and $\delta(x, x)=0$ for all $x$ and $y$, but otherwise place no restrictions on what the error function might look like. For example, in the case of Bloom filters we use a very simply error function: $\delta(x, y)=1$ for any $x \neq y$.

The two correctness notions are given by the experiments in Figure 4. One corresponds to cases where the representations of the true data are public (ERR-Pub) and the other to where they are private (ERR-Priv). We will describe the former and then give a brief explanation of how the latter differs, as the two are closely related to each other.

Both experiments aim to capture the total weight of the errors caused by the adversary's queries. However, because we consider mutable data objects and representations, we only give the adversary credit for Qry calls that produce errors in the "current" data objects $\mathcal{S}_{i}$ and their representations repr $_{i}$. Because we consider mutable data objects and representations, the notion of "current" is defined by calls to the Rep and Up oracles. In the case of Bloom filters, for example, we want to keep track of all the false positives which have been found so far, except for those false positives which have since been turned into true positives.

To track errors, both experiments maintain an array err ${ }_{i}[]$ for every data object $\mathcal{S}_{i}$ that has been defined. Initially, err $i[]$ is implicitly assigned the value of $\perp$ at every index. (We will silently adopt the same convention for all uninitialized arrays.) For purposes of value comparison, we adopt the convention that $\perp<n$ for all $n \in \mathbb{R}$. Now, the array err $i$ is indexed by query functions qry, and the value of $e r r_{i}$ [qry] is the weight of the error caused by qry, with respect to the current data object $\mathcal{S}_{i}$ and current representation repr ${ }_{i}$ (of $\mathcal{S}_{i}$ ). The value of $e r r_{i}$ [qry] is updated within the Qry- and Uporacles, but observe that $e r r_{i}[$ qry] $=\perp$ until ( $i$, qry) is queried to the Qry-oracle. Intuitively, a representation repr $_{i}$ of data object $\mathcal{S}_{i}$ cannot surface errors until it is queried. 


\begin{tabular}{|c|c|c|c|}
\hline $\mathbf{E x p}^{\text {err-pub }}(A) \mathbf{E x p}^{\text {err-priv }}(A)$ & \multirow{8}{*}{$\begin{array}{l}\text { oracle } \operatorname{Rep}(\mathcal{S}): \\
\operatorname{repr} \leftarrow \operatorname{REP}_{K}(\mathcal{S}) \\
\text { if repr }=\perp \operatorname{return} \perp \\
c t \leftarrow c t+1 \\
\operatorname{repr}_{c t} \leftarrow \operatorname{repr} \\
\mathcal{S}_{c t} \leftarrow \mathcal{S} \\
\mathrm{rv} \leftarrow \operatorname{repr} \\
\text { return } \mathrm{rv}\end{array}$} & \multirow{8}{*}{ 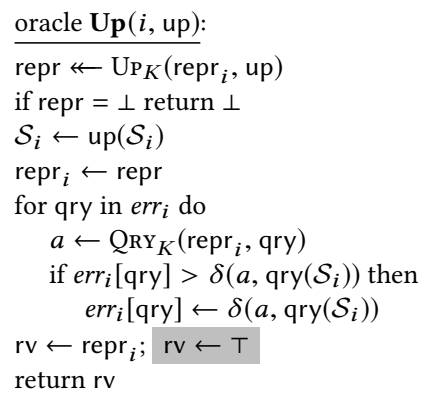 } & \multirow{8}{*}{ 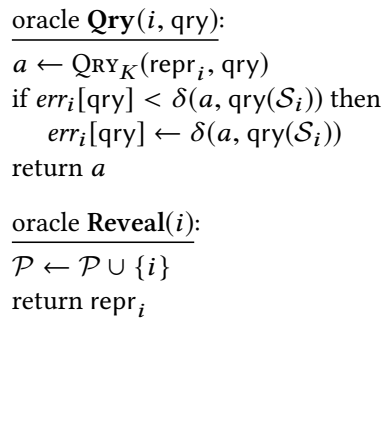 } \\
\hline & & & \\
\hline $\mathcal{P} \leftarrow \emptyset ; c t \leftarrow 0 ; K \leftarrow \mathcal{K}$ & & & \\
\hline$i \leftarrow A^{\text {Rep }, \text { Up }, \text { Qry, Reveal }}$ & & & \\
\hline if $i \in \mathcal{P}$ then return 0 & & & \\
\hline return $\left[\sum_{\text {qry }} \operatorname{err}_{i}[\mathrm{qry}] \geq r\right]$ & & & \\
\hline oracle $\operatorname{Hash}(X)$ : & & & \\
\hline $\begin{array}{l}\text { if } X \notin \mathcal{X} \text { then return } \perp \\
\text { if } T[X]=\perp \text { then } T[X] \leftarrow \mathcal{Y} \\
\text { return } T[X]\end{array}$ & & & \\
\hline
\end{tabular}

Figure 4: Two notions of adversarial correctness. The ERR-Pub notion captures correctness when the representation is always known to the adversary, while the ERR-Priv notion captures correctness when the representation is secret. When modeling a function $H: \mathcal{X} \rightarrow \mathcal{Y}$ as a random oracle, the Hash oracle is given to $A$, REP, UP and QRY.

When Qry ( $i$, qry) executes, the value in $\operatorname{err}_{i}$ [qry] is overwritten iff the error caused by qry is larger than the existing value of $e r r_{i}$ [qry]. The first time ( $i$, qry) is queried to Qry this is guaranteed, since the minimum possible value output by $\delta$ is 0 . After this, the adversary gets credit only for making a worse error than the one already found. This prevents the adversary from trivially winning by repeatedly sending the same error-producing query to Qry. In our Bloom filter example, if Qry finds that qry is a new false positive, it will set err $i$ [qry] to 1 , showing that an additional error has been produced.

When a query $\mathbf{U p}(i$, up $)$ is made, the oracle first updates the data object $\mathcal{S}_{i}$ and its corresponding representation. Now, for each defined value err $_{i}$ [qry], we re-evaluate the error that would be caused by the previously asked qry WRT the newly updated $\mathcal{S}_{i}$ and repr $_{i}$. If the existing value of $e r r_{i}$ [qry] is larger than the error that qry would cause to WRT the newly updated $\mathcal{S}_{i}$ and repr $_{i}$, then we overwrite $e r r_{i}$ [qry] with the smaller value. Doing so ensures that the array err $i$ does not overcredit the attacker for errors against the current data object and representation. For example, if $x$ was previously found to be a false positive for a Bloom filter, and the adversary then inserts $x$ into the data structure, we set $\operatorname{err}_{i}$ [qry ${ }_{x}$ ] to 0 . Since $x$ is now a true positive rather than a false positive, it should no longer be counted as an error.

ERR-Priv differs from ERR-Pub only in that the Rep and Up oracles do not reveal the representation to the adversary. This models the case where the data structure is stored privately, where the adversary can ask queries but not see the full representation. To model the possibility that information about a representation is eventually leaked, we also give the adversary a Reveal oracle that reveals a given representation. However, to prevent this from being trivially equivalent to the public-representation case we do not allow the adversary to win by finding errors in a representation which has been revealed using Reveal. Since the ERR-Pub adversary gets access to the same information that ERR-Priv does without the need for Reveal calls, ERR-Pub security is a stronger notion than ERR-Priv security.

We define the advantage of an ERR-Pub-adversary $A$ as

$$
\operatorname{Adv}_{\Pi, \delta, r}^{\text {err-pub }}(A)=\operatorname{Pr}\left[\operatorname{Exp}_{\Pi, \delta, r}^{\text {err-pub }}(A)=1\right]
$$

\begin{tabular}{|ll|}
\hline$\frac{\operatorname{REP}_{K}^{R}(\mathcal{S})}{Z \leftarrow\{0,1\}^{\lambda} / / \text { Choose a salt } Z}$ & $\frac{\operatorname{QRY}_{K}^{R}\left(\langle M, Z, c\rangle, \mathrm{qry}_{x}\right)}{X \leftarrow B_{m}\left(R_{K}(Z \| x)\right)}$ \\
$\operatorname{repr} \leftarrow\left\langle 0^{m}, Z, 0\right\rangle$ & $\operatorname{return} M \wedge X=X$ \\
for $x \in \mathcal{S}$ do & $\mathrm{UP}_{K}^{R}\left(\langle M, Z, c\rangle\right.$, up $\left._{x}\right)$ \\
$\quad$ repr $\leftarrow \mathrm{Up}_{K}^{R}($ repr, up $x)$ & if $c \geq n$ then return $\perp$ \\
$\quad$ if repr $=\perp$ then return $\perp$ & $M \leftarrow M \vee B_{m}\left(R_{K}(Z \| x)\right)$ \\
return repr & return $\langle M, Z, c+1\rangle$ \\
& \\
\hline
\end{tabular}

Figure 5: Keyed structure $\mathrm{BLOOM}[R, n, \lambda]=\left(\mathrm{ReP}^{R}, \mathrm{QRY}^{R}, \mathrm{UP}^{R}\right)$ is used to define Bloom filter variants used to represent sets of at most $n$ elements. The parameters are a function $R: \mathcal{K} \times\{0,1\}^{*} \rightarrow[\mathrm{m}]^{k}$ and integers $n, \lambda \geq 0$. A concrete scheme is given by a particular choice of parameters. The function $B_{m}$ is defined in Section 2.1.

and write $\mathbf{A d v} \mathbf{v}_{\Pi, \delta, r}^{\text {err-pub }}\left(t, q_{R}, q_{T}, q_{U}, q_{H}\right)$ as the maximum advantage of any ERR-Pub-adversary running in $t$ time steps and making $q_{R}$ calls to Rep, $q_{T}$ calls to Qry, $q_{U}$ calls to $\mathbf{U p}$, and $q_{H}$ calls to Hash in the ROM. We define ERR-Priv advantage in kind, except that we add an extra parameter $q_{V}$ representing the number of calls to Reveal. We sometimes use ERR-Pub1 or ERR-Priv1 to refer to the restriction of the ERR-Pub or ERR-Priv games to the case of $q_{R}=1$; for these we remove the $q_{R}$ parameter from the advantage function.

\section{BLOOM FILTERS}

In this section we consider two classes of Bloom filters, each employing a different strategy to determine when the filter reaches full capacity. The first class is specified in Figure 5. This class of $n$-capped filters captures the classical setting in which the filter is used to represent some fixed number of elements $n \geq 0$. Our construction $\mathrm{BLOOM}[R, n, \lambda]=\left(\mathrm{ReP}^{R}, \mathrm{QRY}^{R}, \mathrm{UP}^{R}\right)$ has two additional parameters besides the cap: a function $R: \mathcal{K} \times\{0,1\}^{*} \rightarrow[m]^{k}$ and the salt length $\lambda \geq 0$. Let $H:\{0,1\}^{*} \rightarrow[m]^{k}$ be a hash function and let $\ell, n, \lambda \geq 0$ be integers. The standard Bloom filter is the structure $\mathrm{BF}[H, n]=\operatorname{BLOOM}\left[\mathrm{ID}^{H}, n, 0\right]$, which we will term the basic Bloom filter. It has no key (the key space of ${ }_{\mathrm{ID}}{ }^{H}$ is $\{\varepsilon\}$, see Section 2.1) and does not use a salt. The salted Bloom filter 
$\operatorname{SBF}[H, n, \lambda]=\operatorname{BLOOM}\left[\operatorname{ID}^{H}, n, \lambda\right]$ is the same except that it allows a nonempty salt. Finally, we consider a salted variant that uses a $\mathrm{PRF}$ instead of a hash function. The keyed Bloom filter $\operatorname{KBF}[F, n, \lambda]$ is the structure $\operatorname{BLoom}[F, n, \lambda]$, where $F: \mathcal{K} \times\{0,1\}^{*} \rightarrow[m]^{k}$ is a PRF. Note that the basic and salted BFs have key spaces $\{\varepsilon\}$ and the keyed BF has key space $\mathcal{K}$.

In this section, we will show that the basic Bloom filter construction $\mathrm{BF}[H, n]$ insecure in our setting. It allows the adversary to make an offline attack that has a high probability of success while using a minimal number of queries. In the immutable setting, where the adversary is constrained to never use the Up oracle, i.e. $q_{U}=0$, it suffices to use the SBF construction in order to provide a security guarantee in either the public-representation or private-representation settings. However, in the case where we allow $q_{U}>0$ so that the adversary can make updates, we will find that SBF is only secure in the ERR-Priv setting. To provide ERR-Pub security when updates are needed, KBF must be used instead.

At the end of the section, we discuss the second class of filters that we call the $\ell$-thresholded. Instead of rejecting updates after a pre-determined number of elements are added to the set, for these we decide the filter is full once at least $\ell \geq 0$ bits of the filter are set. In the usual, non-adaptive setting, this strategy performs similarly to the standard Bloom filter, but we find that a filter threshold allows us to obtain better bounds. We will demonstrate this for salted BFs in the ERR-Priv setting.

NON-ADAPTIVE FALSE-POSITIVE PROBABILITY. Let $\rho:\{0,1\}^{*}[\mathrm{~m}]^{k}$ be a function, $\lambda \geq 0$ be an integer, and define $\operatorname{BLoom}\left[\mathrm{ID}^{\rho}, n, \lambda\right]=$ $\left(\mathrm{REP}^{\rho}, \mathrm{QRY}^{\rho}, \mathrm{UP}^{\rho}\right)$ as in Figure 5. (Note the mild abuse of notation

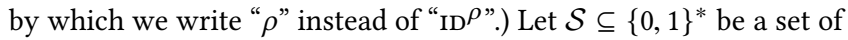
length $n$. We define the non-adaptive, false positive probability for Bloom filters as

$$
\begin{aligned}
P_{k, m}(n)=\operatorname{Pr}[\rho & \leftarrow \operatorname{Func}\left(\{0,1\}^{*},[m]^{k}\right) ; \operatorname{repr} \leftarrow \operatorname{ReP}^{\rho}(\mathcal{S}) ; \\
x & \left.\leftarrow\{0,1\}^{*} \backslash \mathcal{S}: \operatorname{QRY}^{\rho}\left(\operatorname{repr}, \operatorname{qry}_{x}\right)=1 \mid \operatorname{repr} \neq \perp\right] .
\end{aligned}
$$

That is, $P_{k, m}(n)$ is the probability that some $x$ is a false positive for the representation of some $\mathcal{S}$ for which $|\mathcal{X}|=n$ and $x \notin \mathcal{S}$, when a random function is used for hashing. Because of the randomization provided by $\rho$, this probability is independent of $\mathcal{S}$ and $x$. Finding a tight, concrete upper bound for $P_{k, m}(n)$ has proven challenging, but we do understand its asymptotic behavior. Kirsch and Mitzenmacher [20] prove that, for certain choices of $k$ and $m$ as functions of $n$, it holds that $P_{k, m}(n)=\lim _{n \rightarrow \infty}\left(1-e^{-k n / m}\right)^{k}$. Moreover, they demonstrate via simulation that this is a very good approximation of the false positive probability. In lieu of a concrete upper bound, we will refer to $P_{k, m}(n)$ as defined in Equation (1) in the remainder.

ERROR FUNCTION FOR SET-MEMBERSHIP QUERIES. Throughout this section we will use the error function $\delta$ defined as

$$
\delta(x, y)= \begin{cases}0 & \text { if } x=y \\ 1 & \text { otherwise }\end{cases}
$$

This simply indicates whether the query result matched the correct response.

\subsection{Insecurity of unsalted BFs}

The performance of basic Bloom filters is well-understood assuming the choice of set $\S$ being represented is independent of the choice of hash function. When this assumption is violated, however, their performance can be substantially degraded [18]. Here we show that, even when we (optimistically) model the hash function as a random oracle, basic BFs cannot achieve security in our setting. The basic Bloom filter has no salt and no secret key. Let $H:\{0,1\}^{*} \rightarrow[m]^{k}$ be a function, fix $n \geq 0$, and let $\Pi=\mathrm{BF}[H, n]=\left(\mathrm{ReP}^{H}, \mathrm{QRY}^{H}, \mathrm{UP}^{H}\right)$ as defined above. With no per-representation randomness and no secret key to be concealed from the adversary, there is no difference between ERR-Pub and ERR-Priv security, as the adversary can easily compute the representation of any set for itself. This ability of the adversary to reconstruct the set without making queries allows for various attacks that badly harm the accuracy of the filter.

Pollution ATTACKs. Gerbet et al. [18] provide the following example of an attack setting and a potential attack against Bloom filters. Suppose the adversary is interacting with a system representing a dataset with $\Pi$ and that it is able to choose some fraction of the input data. For example, consider a web crawler which performs a "crawl" of webpages [10], following the links on each page it visits in order to index, archive, or otherwise analyze websites. In order to keep track of the set of webpages which have already been visited during a crawl, some crawlers use a Bloom filter which is updated to include each new page the crawler visits. Suppose the adversary controls at least one such webpage along the crawl's path and wishes to deny the spider access to a different webpage, the 'target webpage'. The adversary can choose the links present on its own webpage, which will cause the spider to visit the chosen webpages and set the corresponding bits of its Bloom filter to 1. If those links are chosen in such a way that they produce a false positive for the target webpage, the spider will then erroneously believe it has already visited the target webpage. The target webpage will therefore never be visited during the spider's crawl.

In cases where the adversary is able to control at least some of the filter inputs, the authors describe an attack where the adversary chooses a set of inputs that maximizes the number of $1 \mathrm{~s}$ in the filter. This strategy is especially effective when the structure of the hash function is known to the adversary. In particular, as long as the choice of hash function and any associated parameters are public, the adversary can compute the hash function on its own in order to determine which choices will set the maximum number of bits to 1 , or which choices will set certain target bits to 1 in order to cause specific false positives. They show that with $m=3200$ and $k=4$, the adversary can double the false positive rate if they control 200 out of a total of $n=600$ insertions, under the assumption that $H$ is arbitrary but known to and computable by the attacker.

Gerbet et al. suggest various ways to mitigate pollution attacks, such as choosing the parameters $k, m$, and $n$ so that even if a pollution attack occurs, the false positive rate is kept below some threshold of acceptability. This strategy is potentially viable, but may significantly increase the amount of memory required to store the data structure. The bounds we provide show how the parameters of a filter can be tweaked to keep the error rate low not just in the presence of this specific type of attack, but in the presence of 
any adversary covered by our more general attack model; doing so, however, will require altering the structure.

The authors also discuss the possibility of using a secretlykeyed hash function. In the attack model they consider, where representations are kept private indefinitely, this suffices to prevent the pollution attack they describe. However, under the more general attack models where the representation may eventually be recovered (in the private-representation setting via Reveal) or is public, simply using a PRF without per-representation randomness does not suffice for security in our setting.

TARgET-SET COVERAgE ATTACKs. Of course, exhibiting a high false positive rate is not the only way a Bloom filter might fail to be correct. In particular, it would be undesirable if the filter were consistently incorrect on a particular set of inputs. Rather than pollute the filter, the adversary's goal might be to craft a set of legitimate looking inputs that cover some disjoint target set of inputs. This type of attack is nicely captured by our adversarial model. In a target-set coverage attack, the adversary is given a small target set $\mathcal{T} \subseteq\{0,1\}^{*}$ and searches for a cover set $\mathcal{R} \subseteq\{0,1\}^{*}$ such that $\mathrm{QRY}^{H}\left(\operatorname{ReP}^{H}(\mathcal{R})\right.$, qry $\left._{x}\right)=1$ for each $x \in \mathcal{T}$. Once a suitable cover set is found, the adversary queries $\operatorname{Rep}(\mathcal{R})$. Then for each $x \in \mathcal{T}$, it asks $\operatorname{Qry}\left(\operatorname{qry}_{x}\right)$, achieving a score of $r=|\mathcal{T}|$.

This ERR-Priv1 attack succeeds with probability 1 assuming a covering set can be found. If $|\mathcal{T}| \leq|\mathcal{R}|$, then such a set exists; but finding it may be computationally infeasible, depending on the size of the cover set, the size of the target set, and the parameters of the Bloom filter. In Appendix A we demonstrate that target-set coverage attacks are feasible for practical BF parameters. We do so by simulating the attack when $H$ is a random function (i.e., for each distinct input we choose $k$ integers from $[m]$ at random) for typical choices of $k, m$, and $n$.

\subsection{Salted BFs in the (im)mutable setting}

Here we consider the correctness of Bloom filters when the hashed input is prepended with a salt. Fix $H:\{0,1\}^{*} \rightarrow[m]^{k}$ and $n, \lambda \geq 0$ and let $\Pi=\operatorname{SBF}[H, n, \lambda]$.

If the adversary can update the representation via Up, then it can perform an ERR-Pub attack against $\Pi$ that is closely related to the attacks in the previous section. The adversary calls $\operatorname{Rep}(\emptyset)$, getting an empty filter and the salt in response. It may then use the salt to construct representations on its own just as described in the target-set coverage attack. The works because the adversary can test for errors on its own because it knows the salt. In practice, an adversary may not be able to perform this exact attack, since even in the streaming setting it is possible that the salt is not immediately revealed to the adversary. However, as soon as the adversary does learn the salt, it can immediately launch a pollution attack against the filter, without having to make any queries directly to the filter.

Without the ability to insert elements even after the salt has been seen, the above attack fails. Indeed, when we restrict ourselves to the immutable setting, we can prove the following.
Theorem 4.1 (Immutable ERR-Pub SEcurity of SAlted BFs). Let $p=P_{k, m}(n)$. For all integers $q_{R}, q_{T}, q_{H}, r, t \geq 0$ it holds that

$$
\begin{aligned}
& \operatorname{Adv}_{\Pi, \delta, r}^{\text {err-pub }}\left(t, q_{R}, q_{T}, 0, q_{H}\right) \leq \\
& q_{R} \cdot\left[\frac{q_{H}}{2^{\lambda}}+\left(\frac{p q}{r}\right)^{r} e^{r-p q}\right],
\end{aligned}
$$

where $H$ is modeled as a random oracle, $q=q_{T}+q_{H}$, and $r>p q$.

We consider only the case of $r>p q$ because $p q$ is the expected number of false positives obtained by an adversary that simply uses its knowledge of the salt (after the representation is created) to guess as many random elements as possible. Because this simple adversary can get $p q$ successes on average, we can only hope to provide good security bounds against arbitrary adversaries in the case that $r>p q$.

Before giving the proof, let us take a moment to unpack the result a bit. The bound can be broken down into three main components. The factor of $q_{R}$ means that the bound we can prove is weakened somewhat when a number of representations are observed by the adversary. (In Section 4.4, we will show that we can do better by thresholding rather than capping.) The $q_{H} / 2^{\lambda}$ term corresponds to the probability of the adversary guessing the salt before the representation is constructed, but this will be negligible as long as $\lambda$ is chosen to be sufficiently large (say, $\lambda=128$ ). The final, messier term comes from applying a Chernoff bound to the non-adaptive adversary's probability of succeeding in the experiment given $q=q_{H}+q_{T}$ guesses. By way of clarifying the performance of our bound, we have plotted the last component for various parameters of interest. Let

$$
\zeta_{k, m, n}(q, r)=\left(\frac{p^{*} q}{r}\right)^{r} e^{r-p^{*} q}
$$

where $p^{*}=\left(1-e^{-k n / m}\right)^{k}$, the approximation of the non-adaptive false positive probability given by Kirsch and Mitzenmacher [20]. Figure 6 shows values of $\zeta_{k, m, n}(q, r)$ for varying $m$. What this plot shows is that, for a given error capacity $r$, once a certain lower bound on the filter size is reached, the $\zeta$ term decreases quite quickly. Moreover, the rate at which $\zeta$ decreases scales nicely with the error capacity. For example, if one is willing to tolerate up to $r=10$ false positives for a filter representing $n=100$ elements, then picking a filter length of 3 kilobytes is sufficient to ensure that observing 10 false positives occurs with probability less than $2^{-17}$, even when the adversary can make $q=2^{64}$ Hash or Qry queries.

We concede that requiring a $3 \mathrm{~KB}$ for a filter a set of 100 elements may be prohibitive in some applications. We would require a substantially smaller filter for smaller $q$, but unfortunately, a query complexity of $q=2^{64}$ in the ERR-Pub setting is quite realistic, since the attack can be carried out offline. In the ERR-Priv setting, or in the ERR-Pub setting when we use a PRF instead of a hash function, the adversary's attack is largely online, rendering the $q$ term quite pessimistic. In these settings, a significantly smaller filter will do.

Figure 6 shows filters comparable in size to those discussed in [18], but Bloom filters used in practice are sometimes much larger, on the order of $2^{20}$ or $2^{30}$. We also find benefits in these settings. For example, we can use a $32 \mathrm{~GB}$ filter to store $2^{30}$ elements, each hashed 16 times, in such a way that an adversary making $2^{64}$ queries has a less than $2.2 \times 10^{-3}$ probability of producing 5 or more errors, 


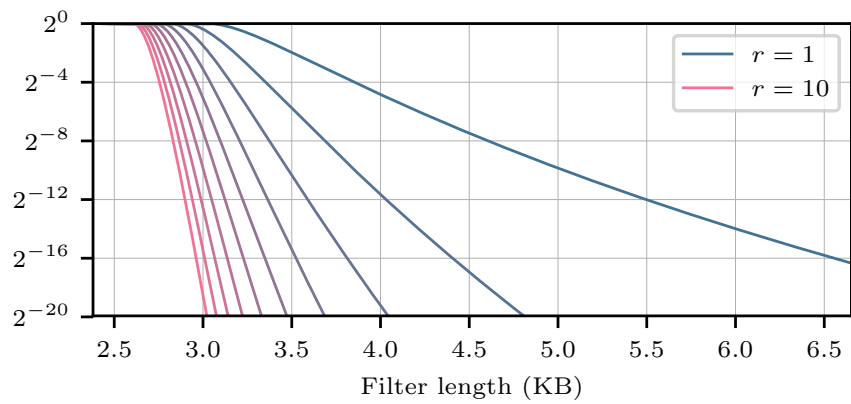

Figure 6: The value of $\zeta_{k, m, n}(q, r)$ (Equation (3)) for $q=2^{64}, k=16$, $n=100$, varying values of $r$ (one line per $r$-value) and filter length $m$ (the $\mathrm{x}$-axis). Note the $\log -2$ scale on the $\mathrm{y}$-axis.

and less than an $8.3 \times 10^{-9}$ probability of producing 10 or more errors. Again, in the ERR-Priv setting or when using a PRF, the number of adversarial queries is likely to be much smaller than this, and so the filter itself can also be made much smaller.

Proof Sketch of Theorem 4.1. The proof makes use of the following lemma for keyless structures, which is proved formally in the full version of the paper.

Lemma 4.2. For every $q_{R}, q_{T}, q_{U}, q_{H}, r, t \geq 0$ and keyless structure $\Gamma$ it holds that

$$
\begin{aligned}
\operatorname{Adv}_{\Gamma, \delta, r}^{\text {err-pub }}\left(t, q_{R}, q_{T}, q_{U}, q_{H}\right) \leq & \\
& q_{R} \cdot \mathbf{A d v}_{\Gamma, \delta, r}^{\text {err-pub1}}\left(O(t), q_{T}, q_{U}, q_{H}\right),
\end{aligned}
$$

The proof is by a fairly straightforward hybrid argument. Because $\Gamma$ is keyless, an ERR-Pub1 adversary can simulate Rep, Up, and Qry calls itself. In the reduction, we choose a random $i \in\left[q_{R}\right]$ and forward only the $i$ th Rep call to our Rep oracle, simulating all other Rep queries. Similarly, Up and Qry calls are forwarded only if they relate to the $i$ th representation, and otherwise are simulated. The ERR-Pub1 adversary wins in at least those cases where the ERR-Pub adversary outputs $i$ and then wins. Since there is a $1 / q_{R}$ chance of the randomly-chosen $i$ matching the ERR-Pub adversary's output we obtain the factor of $q_{R}$ in the bound.

We begin the argument for the full theorem with $A$, an ERR-Pub adversary making 1 query to $\mathbf{R e p}, q_{T}$ queries to Qry, 0 queries to Up, and $q_{H}$ queries to the random oracle Hash. We make the following assumptions, all of which are without loss of generality. First, all of $A$ 's Qry queries follow its Rep query. Second, we assume that $x \notin \mathcal{S}$ for all queries qry ${ }_{x}$ to Qry, where $\mathcal{S}$ was the input to $A$ 's Rep query. This is without loss because Bloom filters admit false positives, but not false negatives. Third, we we assume that $|\mathcal{S}| \leq n$; this is without loss because otherwise Rep outputs $\perp$ and $A$ gets no advantage. Fourth, we assume that all of $A$ 's Hash queries are of the form $Z \| x$, where $|Z|=\lambda$.

We next make a game-playing argument [2], and then obtain the final bound via application of Lemma 4.2. The high-level goal is to rewrite the game so that the probability that one of $A$ 's queries runs up the score is precisely the non-adaptive false positive probability. In other words, our goal is to transition into a setting in which the Bloom filter output by Rep is independent of the outcome of $A$ 's other queries.
The first new game we move to is $\mathbf{G}_{0}$, where the salt $Z$ for the representation of $\mathcal{S}$ is generated prior to executing $B$, and the Hash oracle sets a bad flag if $B$ calls $\operatorname{Hash}(Z \| x)$ before calling Rep. Since this occurs with probability $q_{H} / 2^{\lambda}$, we can bound away the probability of the adversary prematurely guessing the salt with a term of $q_{H} / 2^{\lambda}$, as seen in the final bound.

With this probability established, we can move to a game $\mathbf{G}_{1}$ where the adversary's random oracle is independent of the hash functions used to respond to oracle calls. In this game the adversary's random oracle is incapable of providing useful information.

In game $\mathbf{G}_{2}$, we alter the Hash and Qry oracles so that the winning condition is computed by Hash instead of Qry. The former oracle maintains a table Ans, setting $A n s[x]=\operatorname{QRY}^{H a s h}\left(M, \operatorname{qry}_{x}\right)$ whenever $Z \| x$ is hashed and giving the adversary credit for an error if this QRY does not match with the correct output. The Qry oracle in $\mathbf{G}_{2}$ simply runs Hash and returns $A n s[x]$ when $x$ is queried. We are effectively giving the adversary credit for RO queries that result in false positives for the representation of $\mathcal{S}$, but which it does not explicitly ask of Qry. Note that this change can only increase the adversary's chance of success.

In $\mathbf{G}_{2}$, the adversary may produce an error through either Hash or Qry, so the number of chances the adversary has to produce an error is no greater than $q=q_{H}+q_{T}$. Since the outputs of Hash are uniformly randomly distributed and independent of the adversary's random oracle, the probability of any of these calls producing an error is equal to the non-adaptive error probability for a Bloom filter, $P_{k, m}(n)$. The adversary's chances of success are given by a binomial distribution, to which we can apply a Chernoff bound:

$$
\operatorname{Adv}_{\Pi, \delta, r}^{\text {err-pub1 }}(A)<\frac{q H}{2^{\lambda}}+\left(\frac{p q}{r}\right)^{r} e^{r-p q} .
$$

Applying Lemma 4.2 to move from the single-representation case to the general case, we get our final bound of

$$
\operatorname{Adv}_{\Pi, \delta, r}^{\text {err-pub }}(A) \leq q_{R} \cdot\left[\frac{q H}{2^{\lambda}}+\left(\frac{p q}{r}\right)^{r} e^{r-p q}\right] .
$$

Recall that the attack against mutable salted filters exploited the fact that the adversary learned the salt as soon as the filter was created, and that from this it could compute the hash function on its own. Even if the filter is mutable, we can prevent this attack from working as long as we require that the filter under attack be kept secret from adversaries. In fact, we can attain the following ERR-Priv bound for $\Pi$.

Theorem 4.3 (ERR-Priv SEcUrity of SAlted BFs). Let $p^{\prime}=$ $P_{k, m}(n+r)$. For all integers $q_{R}, q_{T}, q_{U} q_{H}, q_{V}, r, t \geq 0$, if $r>p^{\prime} q_{T}$, then it holds that

$$
\begin{aligned}
\operatorname{Adv}_{\Pi, \delta, r}^{\text {err-priv }}\left(t, q_{R}, q_{T}, q_{U}, q_{H}, q_{V}\right) \leq \\
q_{R} \cdot\left[\frac{q_{H}}{2^{\lambda}}+\left(\frac{p^{\prime} q_{T}}{r}\right)^{r} e^{r-p^{\prime} q_{T}}\right],
\end{aligned}
$$

where $H$ is modeled as a random oracle.

The proof follows a similar structure to that of Theorem 4.1. The main differences come from arguing that without a "lucky" guess of the salt, the adversary cannot use Hash to find false positives, and from having to show that the adversary's access to Up 
does not substantially change the security bound that can be derived. The first of these is straightforward given the private-representation setting, but the second requires investigating how much of an advantage the Up oracle can give, then moving to games where this advantage is taken into account.

Proof Sketch of Theorem 4.3. Just as in the proof of Theorem 4.1, we will assume the adversary just makes a single call to Rep and use Lemma 4.2 to complete the bound. Let $A$ be an ERR-Priv adversary making exactly 1 call to $\mathbf{R e p}, q_{T}$ calls to Qry, $q_{U}$ calls to $\mathbf{U p}$, and $q_{H}$ calls to Hash. Because $A$ creates only a single representation, it will necessarily lose if it calls Reveal on that representation. We may therefore assume without loss of generality that $A$ makes no calls to Reveal.

In addition to the assumptions of Theorem 4.1, we assume without loss of generality that the adversary never uses Up to insert an element into $\mathcal{S}$ which is already present in the set, and never uses $\mathbf{U p}$ to insert an element $x$ where $\operatorname{Qry}\left(\right.$ qry $\left._{x}\right)$ has already been called and has returned a positive result. Since these insertions do not change the filter, the adversary would gain no advantage from performing these updates. Furthermore, we assume without loss of generality that an adversary halts as soon as it determines it has accumulated enough errors to win the experiment.

Since we are seeking a stronger bound, we now wish to isolate the possibility that the adversary ever guesses the salt, as opposed to just guessing the salt before calling Rep. This is no longer a trivial task for the adversary because the representations are private, and so Rep does not directly reveal the salt. We therefore set a bad flag whenever the adversary manages to guess the salt. However, since the adversary is still limited to a total of $q_{H}$ Hash queries, regardless of when the queries are made, we can follow nearly the same argument as in the previous proof to bound away the probability of the bad flag being set at $q_{H} / 2^{\lambda}$. This is the same probability because the probability of the adversary guessing the salt at any time in this experiment is equal to the probability of the adversary guessing the salt in advance (before Rep is called) in the previous game, and the previous game's adversary could choose to make all of its random oracle queries before calling Rep.

Once we have done this, we have a game $\mathbf{G}_{1}$ where the adversary's Hash queries are independent of the Hash queries made by the oracle: it is irrelevant whether the adversary guesses the salt. By our starting assumptions, the same element is never inserted twice. We then take a general adversary $A$ for $\mathbf{G}_{1}$ and construct $B$ that mimics $A$ but making new queries in place of repeat queries. Since such an adversary is at least as likely to win, we can assume without loss of generality that an adversary never queries the same element twice, either. We then move from $B$ to $C$, which behaves the same except that upon finding a true negative using Qry, it immediately calls Up to add the queried element to the data structure. Once $C$ runs out of Up queries, it simply ignores further $\mathbf{U p}$ calls from the simulated $B$.

By our earlier assumptions, there are only two types of update $B$ may make:

(1) Inserting an element which is not already in $\mathcal{S}$ and has previously been tested with Qry, returning a negative result.

(2) Inserting an element which is not already in $\mathcal{S}$ and has not previously been tested with Qry.
The effects of type 1 updates on the representation are identically distributed, as are the effects of type 2 updates on the representation. However, the effects of the two types of update are not identically distributed compared to each other. In particular, making a type 1 update ensures that at least one new bit in the filter will be set to 1 , since the distribution of $B_{m}(\operatorname{Hash}(Z \| x))$ is conditioned on not producing a false positive. On the other hand, making a type 2 update provides no guarantee about how many bits in the filter might be set to 1 . Type 1 updates are therefore always preferable for an adversary attempting to produce false positives.

All of the updates made by $C$ but not by $B$ are type 1 updates, which are maximally effective at increasing the error rate. Therefore any Qry calls made by $B$ have at least the same probability of causing an error when made by $C$, and so $C$ has at least the same success rate with the same resources.

We next change to a game which automatically inserts $x$ into $\mathcal{S}$ after computing the correct response to the query, but which does not remove credit for inserting a false positive. Since false positives being inserted does increment the total number of elements being represented, to ensure that the adversary is not penalized in this game we increase the maximum number of elements that can be inserted from $n$ to $n+r$.

We can then bound the adversary's chances of success with another binomial distribution, where the adversary has $q_{T}$ chances and the probability of a success is identical to that with a Bloom filter containing $n+r$ elements. Applying a Chernoff bound, we get:

$$
\operatorname{Pr}\left[\mathbf{G}_{2}(E)=1\right] \leq e^{r-p^{\prime} q_{T}}\left(\frac{p^{\prime} q_{T}}{r}\right)^{r} .
$$

Again we apply Lemma 4.2 to get a final bound of

$$
\operatorname{Adv}_{\Pi, \delta, r}^{\text {err-priv }}(A) \leq q_{R} \cdot\left[\frac{q_{H}}{2^{\lambda}}+\left(\frac{p^{\prime} q_{T}}{r}\right)^{r} e^{r-p^{\prime} q_{T}}\right] .
$$

\subsection{Keyed BFs}

Salted BFs are ERR-Priv secure in general, and are ERR-Pub secure in the immutable setting, but are not ERR-Pub secure when the adversary has access to an Up oracle. Our argument for the ERR-Priv security of salted Bloom filters is made possible by virtue of the structure under attack not being revealed to the adversary. While this is realistic in many applications, it may be desirable for the Bloom filter to be public and updatable. Here we show that building a Bloom filter from a PRF suffices for security in this setting. Let $F: \mathcal{K} \times\{0,1\}^{*} \rightarrow[m]^{k}$ be a function, fix integers $n, \lambda \geq 0$, and let $\Pi=\operatorname{KBF}[F, n, \lambda]$.

TheOrem 4.4 (ERR-Pub SECURITy OF Keyed BFs). Let $p^{\prime}=$ $P_{k, m}(n+r)$. For integers $q_{R}, q_{T}, q_{U}, q_{H}, r, t \geq 0$ such that $r>p^{\prime} q_{T}$, it holds that

$$
\begin{gathered}
\operatorname{Adv}_{\Pi, \delta, r}^{\mathrm{err}-\mathrm{pub}}\left(t, q_{R}, q_{T}, q_{U}, q_{H}\right) \leq \\
\mathbf{A d v}_{F}^{\mathrm{prf}}\left(O(t), n q_{R}+q_{T}+q_{U}\right)+\frac{q_{R}^{2}}{2^{\lambda}}+\left(\frac{p^{\prime} q_{R} q_{T}}{r}\right)^{r} e^{r-p^{\prime} q_{R} q_{T} .}
\end{gathered}
$$

As usual, our strategy will be to move to the non-adaptive setting via a sequence of game transitions, but the details of how we get there differ from the case of keyless Bloom filters. In particular, 
since we are using a PRF, the initial parts of the proof deal with the adversary potentially being able to break the PRF and with the possibility of the salts repeating rather than with the adversary being able to guess the salt.

Proof Sketch of Theorem 4.4. Unlike in the previous two proofs, we cannot use Lemma 4.2 because an adversary cannot simulate the oracles without knowing the private key. Instead, the first thing we want to do is to bound the probability that the adversary can break the PRF.

The number of times the PRF is evaluated on distinct inputs is bounded by the number of queries available to the adversary. In particular, Qry and Up each call the PRF once, while Rep may call the PRF up to $n$ times. Thus there are at most $Q=q_{T}+q_{U}+n q_{R}$ queries to $\mathbf{F}$. In $\mathbf{G}_{1}$, we have a game which is identical to the original except that it uses random sampling in place of the PRF. If $A$ cannot distinguish the PRF from a random function then these games are indistinguishable from the adversary's perspective. We exhibit a $O(t)$-time, PRF-adversary $D$ making at most $Q$ queries to its oracle. Adversary $D^{\mathbf{F}}$ works by executing $A$ in game $\mathbf{G}_{1}$, except that whenever the game calls its $\mathbf{F}$, adversary $D$ uses its own oracle to compute the response. Finally, when $A$ halts, if the winning condition in $\mathbf{G}_{1}$ is satisfied, then $D$ outputs 1 as its guess; otherwise it outputs 0 . Then conditioning on the outcome of the coin flip $b$ in $D$ 's game, we have that $\operatorname{Adv}_{F}^{\operatorname{prf}}(D)=\operatorname{Pr}\left[\mathbf{G}_{0}(A)=1\right]-\operatorname{Pr}\left[\mathbf{G}_{1}(A)=1\right]$. Next, our goal is to argue, in a similar manner as to the previous theorems, that all of the oracle calls are independent. In order to guarantee this we must deal with the possibility of a salt collision between different representations. In $\mathbf{G}_{2}(A)$ we require that all salts be distinct between representations. By the birthday bound, collisions between randomly-generated salts occur with frequency at most $q_{R}^{2} / 2^{\lambda}$, so $\operatorname{Pr}\left[\mathbf{G}_{1}(A)=1\right] \leq q_{R}^{2} / 2^{\lambda}+\operatorname{Pr}\left[\mathbf{G}_{2}(A)=1\right]$.

With guaranteed-unique salts, the result of each Rep, Up, and Qry call for a given representation is independent of the calls for all other representations. By an almost identical argument to the one in the proof of Theorem 4.3, we can reduce from any $A$ to an adversary $B$ which follows any Qry call that finds a true negative with an Up call to insert that element, and therefore move to $\mathbf{G}_{3}(B)$, which as in the Theorem 4.3 proof performs an update after each query is made, with the guarantee that $\operatorname{Pr}\left[\mathbf{G}_{1}(A)=1\right] \leq \operatorname{Pr}\left[\mathbf{G}_{2}(B)=1\right]$.

Finally, we must deal with the possibility that the adversary chooses which representations to target with $\mathbf{U p}$ and Qry calls based on the result of Rep, since some representations may be more full than others. In game $\mathbf{G}_{4}$, we allow the adversary credit if a call to Qry produces an error in any of the representations that have been constructed. Furthermore, the updates made by Qry apply to all representations that are not already full. Since all Up calls are identically and independently distributed, and having more elements in a filter cannot decrease the false positive rate, the fact that some representations may become full more quickly than they otherwise would have can only help the adversary.

We come again in a situation where we can apply the standard, non-adaptive error bound, using the same sort of bound for the binomail distribution:

$$
\operatorname{Pr}\left[\mathbf{G}_{4}(B)=1\right] \leq e^{r-p^{\prime} q_{R} q_{T}}\left(\frac{p^{\prime} q_{R} q_{T}}{r}\right)^{r} .
$$

\begin{tabular}{|c|c|}
\hline $\operatorname{REP}_{K}^{R}(\mathcal{S})$ & $\operatorname{QRY}_{K}^{R}\left(\langle M, Z\rangle, \operatorname{qry}_{x}\right)$ \\
\hline 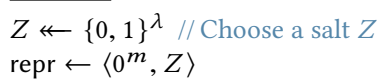 & 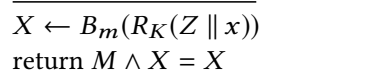 \\
\hline $\begin{array}{l}\text { for } x \in \mathcal{S} \text { do } \\
\left.\quad \text { repr } \leftarrow \mathrm{Up}^{R} \text { (repr, gry }\right)\end{array}$ & $\mathrm{UP}_{K}^{R}\left(\langle M, Z\rangle\right.$, qry $\left._{x}\right)$ \\
\hline $\begin{array}{l}\text { if repr }=\perp \text { then return } \perp \\
\text { return repr }\end{array}$ & $\begin{array}{l}\text { if } w(M)>\ell \text { then return } \perp \\
\text { return }\left\langle M \vee B_{m}\left(R_{K}(Z \| x)\right), Z\right\rangle\end{array}$ \\
\hline
\end{tabular}

Figure 7: The class of $\ell$-thresholded Bloom filters is given by $\operatorname{BLOOM}_{\mathrm{ft}}[R, \ell, \lambda]=\left(\mathrm{ReP}^{R}, \mathrm{QRY}^{R}, \mathrm{UP}^{R}\right)$. This is a slight variant of $n$ capping wherein we use the Hamming weight of the filter $(w$, as defined in Section 2.1) to decide if the filter is full.

So, substituting this bound back into the earlier advantage inequalities, we find the final bound of

$$
\begin{aligned}
& \operatorname{Adv}_{\Pi, \delta, r}^{\text {err-pub }}(A) \leq \\
& \quad \operatorname{Adv}_{F}^{\operatorname{prf}}(D)+\frac{q_{R}^{2}}{2^{\lambda}}+\left(\frac{p^{\prime} q_{R} q_{T}}{r}\right)^{r} e^{r-p^{\prime} q_{R} q_{T}} .
\end{aligned}
$$

The fact that both a key and a salt are used in the KBF construction is critical. In particular, without the per-representation randomness given by the salt, we would not be able to argue that Up and Qry calls are independent across representations. On the contrary, seeing the representation of a singleton set $\{x\}$ would immediately allow the adversary to test whether $x$ was a member in every other representation that had been constructed, simply by testing whether every bit set to 1 in the representation of $\{x\}$ was also set to 1 in other representations. Even in the ERR-Priv game, using the Reveal oracle on some representations leaks information about other representations, and again we cannot use the argument that provides the above bound.

We note that Gerbet et al. [18] suggest using keyed hash functions as one possibility for constructing secure filters, which is equivalent in our terminology to using a keyed but unsalted filter. The distinction is that Gerbet et al. assume that representations are kept private indefinitely, an assumption similar to that underlying our ERR-Priv game, but with the stronger restriction that the adversary has no equivalent of a Reveal oracle. This makes their notion of security much weaker than ours with respect to keyed structures.

\section{4 $\ell$-thresholded BFs}

So far we have proven bounds for only $n$-capped BFs. It is important to understand the security of this class of structures because it is representative of how BFs are used in practice. In this section we demonstrate show that we improve security bounds by defining "fullness" in terms of the weight of the filter, rather than the number of elements it represents. The general form of this alternate construction is formally defined in Figure 7 . We can define the more specific constructions $\mathrm{BF}_{\mathrm{ft}}[H, \ell], \mathrm{SBF}_{\mathrm{ft}}[H, \ell, \lambda]$, and $\mathrm{KBF}_{\mathrm{ft}}[H, \ell, \lambda]$ in an exactly the same way as the $n$-capped variants. Here we only consider case of $\Pi=\operatorname{SBF}_{\mathrm{ft}}[H, \ell, \lambda]$ and compare it to the SBF construction in Section 4.2. The non-adaptive false positive probability is similar is similar to capped filters, since the number of 1 bits in 
the filter can be closely predicted from the number of elements in a randomly-selected underlying set. Because of this, and because we are able to demonstrate better security bounds for an $\ell$-thresholded filter than for a capped filter, we suggest this as a way of providing strong security guarantees for even smaller filter sizes.

Theorem 4.5 (ERR-Priv SECURITY OF THRESholded BFs). Let $p_{\ell}=((\ell+k) / m)^{k}$. For integers $q_{R}, q_{T}, q_{U}, q_{H}, q_{V}, r, t \geq 0$ such that $r>p_{\ell} q_{T}$, it holds that

$$
\begin{aligned}
\operatorname{Adv}_{\Pi, \delta, r}^{\text {err-priv }}\left(t,, q_{R}, q_{T}, q_{U}, q_{H}, q_{V}\right) & \leq \\
& \frac{q_{R}\left(q_{H}+q_{R}\right)}{2^{\lambda}}+e^{r-p_{\ell} q_{T}}\left(\frac{p_{\ell} q_{T}}{r}\right)^{r},
\end{aligned}
$$

where $H$ is modeled as a random oracle.

From a technical point of view, the main difference between thresholded and capped filters is that attacks cannot set more than $\ell+k$ bits of the filter to 1 , regardless of how the attack is conducted. The thrust of the proof is to assume the adversary will always be able to produce such a maximally full filter, and then use a standard binomial-distribution-based bound to place a limit on the adversarial advantage even in this worst-case scenario.

Proof Sketch of Theorem 4.5. As before, we assume without loss of generality that there are no insertions of or queries for elements of $\mathcal{S}$. To avoid the unfortunate $q_{R}$ factor in the bound, we do not make use of Lemma 4.2 in this proof. Because of that, we must find some other way to ensure that Reveal is not useful to the adversary. In particular, if there are unique salts across representations, the Rep, Qry, and Up calls for one representation will be independent of those for other representations, since the unique salt is passed as part of the input. Therefore in $\mathbf{G}_{1}$ we specify that all salts created will be unique, but deny access to Reveal. By the birthday bound, the probability of salts repeating in the original game is no more than $q_{R}^{2} / 2^{\lambda}$. Since Reveal still does not help the adversary, we can also remove the adversary's access to the Reveal oracle in $\mathbf{G}_{1}$.

Next, we want to ensure that the adversary's direct Hash queries are independent of those made indirectly through Rep, Qry, and Up. Since Hash uses random sampling to fill a shared table, this occurs if and only if the adversary hashes $Z_{i} \| x$ for some salt $Z_{i}$ used by one of the representations created by Rep. By an argument very similar to that in the previous proofs, the adversary has at most $q_{H} / 2^{\lambda}$ probability of doing this for each representation. However, since there are now $q_{R}$ representations, each with a distinct salt, there is at most a $q_{R} q_{H} / 2^{\lambda}$ probability of the adversary correctly guessing a salt. We therefore add a bad flag which is set if the adversary guesses a salt in this manner, and bound away the probability as $q_{R} q_{H} / 2^{\lambda}$.

Now the adversary derives no advantage from guessing the salts of the representations, in the sense that the outputs of Hash are independent of the results of the outputs of Rep, Qry, and Up The next oracle we want to target is Up. Now that we have a filter threshold, we want to argue that the adversary cannot use $\mathbf{U p}$ to mount an effective attack. To do this, we move to a game where the $\mathbf{R e p}$ oracle creates the filter as normal and then randomly sets bits until filter is full (i.e., its Hamming weight is at least $\ell$ ), which is $\ell+k$ (since updates are not allowed when more than $\ell$ bits are set, and a single update may set at most $k$ bits to 1 ). Due to the independence results for the different oracles, we can argue that this does not decrease the adversary's probability of winning.

We next change the game so that calls do not actually change the representation. Since each representation is created using independent Hash outputs and then filled in a uniform random manner until $\ell+k$ bits are set to 1 , and is never modified afterwards, the representations themselves are random bitmaps which are uniformly distributed over the set of $m$-length bitmaps with $\ell+k$ bits set to 1. This allows us to move a game where the adversary is given a single arbitrary bitmap repr of length $m$ with $\ell+k$ bits set to 1 and makes Qry calls exclusively for repr, winning if it produces $r$ errors for that 'representation'. Since the hashes used to construct the representation are independent of the hashes used to query the representation, this does not decrease the adversary's probability of winning.

Since Qry calls with distinct inputs have independent outputs, each Qry call made by $D$ has the same probability of producing a false positive. In particular, the probability of any one of the $k$ hashes colliding with a 1 bit is $(\ell+k) / m$, and the probability of all $k$ outputs doing so is then $((\ell+k) / m)^{k}$. If we let $\mathcal{X}$ be the set of all inputs made to Qry, we again have a binomial distribution where $q_{T}$ queries are made. We can once more apply a Chernoff bound, as long as $p_{\ell} q_{T}<r$, to get the final bound of:

$$
\operatorname{Adv}_{\Pi, \delta, r}^{\text {err-priv }}(A) \leq \frac{q_{R}\left(q_{H}+q_{R}\right)}{2^{\lambda}}+e^{r-p_{\ell} q_{T}}\left(\frac{p_{\ell} q_{T}}{r}\right)^{r} .
$$

\subsection{Discussion}

These results show that the standard Bloom filter construction is weak to adaptive adversaries. Moving to the salted SBF construction mitigates this, but if filters are public they must be both large and immutable. In the ERR-Priv setting updates do not break security and the minimum size of the filter to guarantee a fixed error rate is considerably reduced. The guarantee (or, equivalently, filter size) can be further improved, especially if the number of representations constructed is large, by using $\ell$-thresholding. Additionally, if the filters themselves cannot be kept private but a secret key for the hash functions can be concealed from adversaries, the KBF construction shows how to provide security in the ERR-Pub setting.

These requirements are more stringent than the mitigations suggested by Gerbet et al. [18] due to our stronger attack model (where multiple filters can be constructed, and sometimes revealed, to the adversary) and our goal of establishing a general security bound for any adversary rather than mitigating specific attacks. If $q_{R}$ is small, our ERR-Priv guarantee for SBF and ERR-Pub guarantee for KBF show that filters need not be made much larger than Gerbet et al.'s in order to provide comparable security against more general adversaries. If $q_{R}$ is large, however, the $q_{R}$ term in the error bounds means that the filters must be made large to provide good error guarantees. In this scenario, however, the $\ell$-thresholding class of filter provides a way to get strong error guarantees without significantly increasing the filter size.

COMPARISON TO $n$-CAPPING. Figure 8 shows the dominant terms in the ERR-Priv bounds for $n$-capped and $\ell$-threholded salted BFs (Theorem 4.3 and 4.5 respectively). This shows us that the bounds 


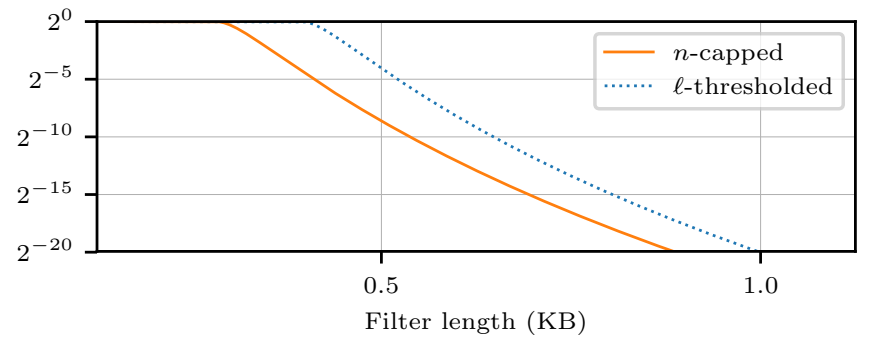

Figure 8: Performance of $n$-capped versus $\ell$-thresholded Bloom filters. The solid orange line shows the value of $\zeta_{k, m, n}(q, r)$ for $k=16$, $n=100, q=2^{16}, r=1$, and varying $m$ (on the x-axis). The dotted blue line shows the dominate term in the bound of Theorem 4.5 for $\ell=n k$. The bounds are comparable, but thresholding would perform much better than capping for even modest $q_{R}$.

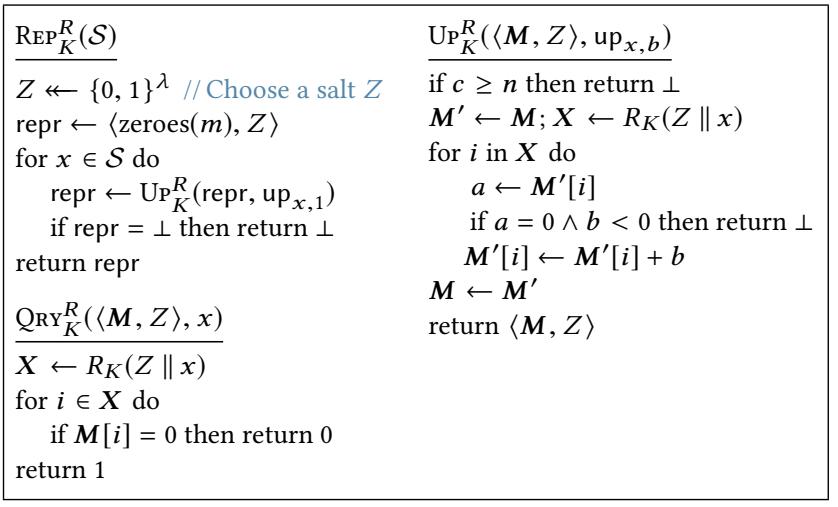

Figure 9: Keyed structure $\operatorname{CoUnT}[R, \ell, \lambda]$ given by $\left(\mathrm{ReP}^{R}, \mathrm{QRY}^{R}, \mathrm{UP}^{R}\right)$ is used to define the $\ell$-thresholded version of a counting filter. The parameters are a function $R: \mathcal{K} \times\{0,1\}^{*} \rightarrow[m]^{k}$ and integers $\ell, \lambda \geq$ 0 . A concrete scheme is given by a particular choice of parameters. The function $w^{\prime}$, used to determine if the filter is full, is defined in Section 2.1.

are comparable for $\ell=n k$, which is a reasonable choice of $\ell$ given that a set of size $n$ can set at most $n k$ bits to 1 , and this upper bound only occurs in the unlikely circumstance that there are no hash collisions during insertion. When we take into account the factor of $q_{R}$ present in the $n$-capped security bound, we conclude that thresholding provides significantly more security if the adversary is allowed a non-negligible number of calls to $q_{R}$.

\section{COUNTING FILTERS}

Counting filters are a modified version of Bloom filters which are designed to, like a count min-sketch, allow for deletion as well as insertion [15]. Unlike CMSes, however, counting filters are designed to handle set membership queries rather than frequency queries. Despite this, the two structures are closely related in terms of security properties. We show that ERR-Pub security is similarly impossible, but employing $\ell$-thresholding allows for ERR-Priv security with a bound that is close to count min-sketch. This $\ell$-thresholded filter is formally defined in Figure 9.
ERROR FUNCTION FOR FREQUENCY QUERIES. Unlike with a Bloom filter or count min-sketch, counting filters must account for two different types of errors: false positives and false negatives. To be as general as possible, we define a parametrized error function $\delta$ for positive $\delta^{+}, \delta^{-} \in \mathbb{R}$ as

$$
\delta(x, y)= \begin{cases}0 & \text { if } x=y \\ \delta^{+} & \text {if } x=1, y=0 \\ \delta^{-} & \text {if } x=0, y=1\end{cases}
$$

This means that false positives are given a weight of $\delta^{+}$while false negatives are given a weight of $\delta^{-}$, and correct responses are given a weight of 0 .

\subsection{Insecurity of public counting filters}

Any counting filter construction necessarily fails to satisfy ERR-Pub correctness for the same reasons as in the case of count min-sketch. In particular, the adversary can call $\operatorname{Rep}(\emptyset)$ to receive an empty representation, insert an element $x$, observe which counters are incremented by this insertion, and then delete $x$. By doing this repeatedly, the adversary can gain information about which elements overlap with which combinations of other elements, and can therefore mount the same attack described in Section 6.1.

\subsection{Private Thresholded Counting Filters}

Theorem 5.1. Fix integers $q_{R}, q_{T}, q_{U}, q_{H}, q_{V}, r, t \geq 0$, let $p_{\ell}=$ $((\ell+1) / m)^{k}$, and let $r^{\prime}=\left\lfloor r / \max \left(\delta^{+}, k \delta^{-}\right)\right\rfloor$. For all such $q_{R}, q_{T}$, $q_{U}, q_{H}, q_{V}, r$, and $t$, if $r^{\prime}>p_{\ell} q_{T}$ then

$$
\begin{aligned}
\operatorname{Adv}_{\Pi, \delta, r}^{\text {err-priv }}\left(O(t), q_{R}, q_{T}, q_{U}, q_{H}, q_{V}\right) \leq \\
q_{R} \cdot\left[\frac{q_{H}}{2^{\lambda}}+e^{r^{\prime}-p_{\ell}} q_{T}\left(\frac{p_{\ell} q_{T}}{r^{\prime}}\right)^{r^{\prime}}\right],
\end{aligned}
$$

where $H$ is modeled as a random oracle.

The proof combines details from the proofs of count minsketch bounds and the proofs of Bloom filter bounds. In particular, we begin by following an argument as in the count min-sketch case to limit the advantage the adversary can get from deleting elements and from re-inserting elements of the original set. There is a difference in the counting filter case in that inserting a known false positive cannot benefit the adversary, while deleting it can. This is the opposite of the count min-sketch case, but the effect on the bound is quite similar. In each case we give the adversary additional credit for finding these false positives while constraining them to not modify false positives they find. This causes $r^{\prime}$ to appear in the proof bound rather than $r$ in each case, but because of the different error functions the definitions of $r^{\prime}$ are slightly different. After this step, we observe that a counting filter without deletion behaves the same as an ordinary Bloom filter in terms of how it responds to queries. We can therefore borrow the arguments used to establish the bounds in Theorem 4.5 to finish the proof.

Proof Sketch of Theorem 5.1. As with the proof of Theorem 4.1, we derive a bound in the ERR-Priv1 case and then use Lemma 4.2 to move from ERR-Priv1 to the more general case of ERR-Priv. Because we are in the ERR-Priv1 case, we may assume without loss of generality that the adversary does not call Reveal, 
since revealing the only representation automatically prevents the adversary from winning.

As in the proof of Theorem 4.1, we first add a $b a d_{1}$ flag that gets set if the adversary ever hashes with the actual salt used by the representation. By a very similar argument, we can move to $\mathbf{G}_{1}$, where the behavior is different only when the $b a d_{1}$ flag is set, which happens with probability at most $q_{H} / 2^{\lambda}$.

Unlike in the case of a count min-sketch, it is entirely possible for deletions to benefit the adversary in this game. In particular, if $x$ is found to be a false positive, deleting $x$ may cause up to $k$ elements of $\mathcal{S}$ to become false negatives. We therefore move to a game $\mathbf{G}_{2}$ where the adversary gets credit for either a single false positive or for $k$ false negatives whenever it finds a false positive, but where the adversary cannot delete any false positives that it finds. We let $r^{\prime}=\left\lfloor r / \max \left(\delta^{+}, k \delta^{-}\right)\right\rfloor$represent the number of false positives the adversary has to find in $\mathbf{G}_{2}$ in order to win. In order to prevent the adversary from being penalized by the filter becoming full too early, we also raise the threshold from $\ell$ to $\ell+r^{\prime}$ in $\mathbf{G}_{2}$. Now for any $A$ for $\mathbf{G}_{1}$, we can construct $B$ for $\mathbf{G}_{2}$ that simulates $A$, keeping track of all query responses and forwarding all oracle queries in the natural way, except that calls to delete false positives are ignored. Since Up never fails for $B$ due to the increased threshold, and since $B$ gets automatic credit for any false negatives that might have been caused by deleting false positives, $B$ succeeds whenever $A$ does, i.e. $\operatorname{Pr}\left[\mathbf{G}_{1}(A)=1\right] \leq \operatorname{Pr}\left[\mathbf{G}_{2}(B)=1\right]$.

Since the remaining deletions do not cause errors, we can use the same argument as in the proof of Theorem 6.1 to reduce from $B$ to an adversary $C$ which does not make deletions at all. In $\mathbf{G}_{3}$, we further reduce from a counting filter to a normal Bloom filter by capping each of the counters in the filter at 1 . Since no deletions are performed, a counter in $\mathbf{G}_{3}(C)$ is nonzero if and only if the same counter in $\mathbf{G}_{2}(C)$ is nonzero. So Qry behaves the same in $\mathbf{G}_{3}$ as it $\operatorname{did}$ in $\mathbf{G}_{2}$, and $\operatorname{Pr}\left[\mathbf{G}_{2}(C)=1\right] \leq \operatorname{Pr}\left[\mathbf{G}_{3}(C)=1\right]$.

Note that $\mathbf{G}_{3}$ is actually simulating an ordinary Bloom filter, since all 'counters' in the filter are restricted to the range $\{0,1\}$, there are no deletions, and any insertions just set the corresponding bits to 1 . In fact, this game is identical to $\mathbf{G}_{2}$ in the proof of Theorem 4.5 except that the adversary need only accumulate $r^{\prime}$ errors instead of $r$ errors and the threshold is $\ell+r^{\prime}$ instead of $\ell$. We can therefore use the same argument to produce the final bound of

$$
\operatorname{Adv}_{\Pi, \delta, r}^{\text {err-priv }}(A) \leq q_{R} \cdot\left[\frac{q_{H}}{2^{\lambda}}+e^{r^{\prime}-p_{\ell} q_{T}}\left(\frac{p_{\ell} q_{T}}{r^{\prime}}\right)^{r^{\prime}}\right] .
$$

\subsection{Discussion}

The results for counting filters are similar to the results for countmin sketch, as might be expected given the similarities in terms of both the supported updates and the structure of the representations themselves (any count-min sketch can be transformed into a counting filter by adding all the rows together element-wise.) In particular, we again see that counting filters which are publicly visible cannot provide good security guarantees. This means that counting filters intended for a security-sensitive setting should be kept hidden from potential adversaries. Furthermore, our bound relies on per-representation random salts and $\ell$-thresholding, so these changes should also be taken into account when constructing

\begin{tabular}{|c|c|}
\hline $\operatorname{ReP}_{K}^{R}(\mathcal{S})$ & $\mathrm{UP}_{K}^{R}\left(\langle\boldsymbol{M}, Z\rangle\right.$, up $\left._{x, b}\right)$ \\
\hline$Z \leftarrow\{0,1\}^{\lambda} / /$ Choose a salt $Z$ & \multirow{9}{*}{$\begin{array}{l}\text { full } \leftarrow \vee_{i \in[1 . . k]}\left[w^{\prime}(M[i])>\ell\right] \\
\text { if } f u l l \text { then return } \perp \\
\boldsymbol{M}^{\prime} \leftarrow M ; X \leftarrow R_{K}(Z \| x) \\
\text { for } i \text { in }[1 . . k] \text { do } \\
\quad a \leftarrow M^{\prime}[i][X[i]] \\
\quad \text { if } a=0 \wedge b<0 \text { then return } \perp \\
\quad M^{\prime}[i][X[i]] \leftarrow a+b \\
M \leftarrow M^{\prime} \\
\text { return }\langle\boldsymbol{M}, Z\rangle\end{array}$} \\
\hline for $i$ in $[1 . . k]$ do & \\
\hline $\boldsymbol{M}[i] \leftarrow \operatorname{zeroes}(m)$ & \\
\hline repr $\leftarrow\langle\boldsymbol{M}, Z\rangle$ & \\
\hline for $x \in \mathcal{S}$ do & \\
\hline repr $\leftarrow \mathrm{Up}_{K}^{R}\left(\right.$ repr, up $\left.{ }_{x, 1}\right)$ & \\
\hline if repr $=\perp$ then return $\perp$ & \\
\hline return repr & \\
\hline $\mathrm{QRY}_{K}^{R}\left(\langle\boldsymbol{M}, Z\rangle, \mathrm{qry}_{x}\right)$ & \\
\hline \multirow{3}{*}{\multicolumn{2}{|c|}{ 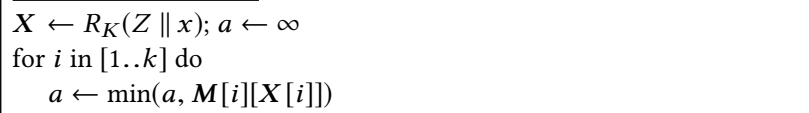 }} \\
\hline & \\
\hline & \\
\hline return $a$ & \\
\hline
\end{tabular}

Figure 10: Keyed structure $\operatorname{SкETCH}[R, \ell, \lambda]$ given by $\left(\mathrm{REP}^{R}, \mathrm{QRY}^{R}, \mathrm{UP}^{R}\right)$ is used to define count min-sketch variants. The parameters are a function $R: \mathcal{K} \times\{0,1\}^{*} \rightarrow[m]^{k}$ and integers $\ell, \lambda \geq 0$. A concrete scheme is given by a particular choice of parameters. The function $w^{\prime}$, used to determine if the sketch is full, is defined in Section 2.1.

secure counting filters. The size increase of the filters is comparable to the size increase of count min-sketches, but is distinct in that it depends on the relative weight of false positives as opposed to false negatives. False negatives impact the bound more than false positives due to the scaling factor of $k$ that appears in $r^{\prime}$, which indicates that applications seeking to minimize false negatives will require larger filters than those seeking to minimize false positives. This is distinctly different than in the non-adaptive setting, where false positives are much more common in counting filters than false negatives, and therefore much more relevant in determining the minimum size of the filter.

\section{COUNT-MIN SKETCHES}

The count min-sketch (CMS) data structure is designed to concisely estimate the number of times a datum has occurred in a data stream. In other words, it is designed to estimate the frequency of each element of a multiset. The data structure is similar to a Bloom filter, but instead of a length- $m$ array of bits it uses a $k$-by- $m$ array of counters. It is designed to deal with streams of data in the nonnegative turnstile model [8], which means the sketch accommodates both insertions and deletions but does not allow any entries to have a negative frequency. Our construction $\operatorname{S\kappa ETcH}[R, \ell, \lambda]$ defined in Figure 10 involves an $\ell$-thresholded variation of this structure. The traditional description of a count min-sketch involves a parameter $n$ describing the size of the input stream, whereas our $\ell$ describes the maximum number of nonzero counters. As in the case of Bloom filters, this does not significantly change the operation of the sketch in a non-adversarial setting, since for random input streams the number of nonzero counters is closely related to the number of elements in the stream. In the presence of an adversary, however, we expect $\ell$-thresholding to provide better security bounds than the traditional definition would provide by handicapping pollution attacks and similar adversarial strategies. 
We will show that, in the ERR-Pub setting, the count minsketch structure is insecure regardless of whether $\ell$-thresholding is used or not, and regardless of the details of the behavior of the function $R$. On the other hand, we show that ERR-Priv security is achievable even under the assumption that a salted but unkeyed hash function is used, i.e. $\lambda>0$ but $\mathcal{K}=\{\emptyset\}$.

Non-ADAPTIVE ERROR BOUND. The CMS is designed to minimize the number of elements whose frequencies are overestimated, while still allowing for reasonably low memory usage. For a function $\rho$ and integer $\lambda \geq 0$, let $\operatorname{SKETCH}\left[\mathrm{ID}^{\rho}, n, \lambda\right]=\left(\operatorname{REP}^{\rho}, \mathrm{QRY}^{\rho}, \mathrm{UP}^{\rho}\right)$ as defined previously. If $\mathcal{S}$ is a multiset containing a total of $n$ elements (counting duplicates as separate elements), i.e. $\mathcal{S} \in \operatorname{Func}\left(\{0,1\}^{*}, \mathbb{N}\right)$ in our syntax, and $x \in\{0,1\}^{*}$ is any string, possibly but not necessarily a member of $\mathcal{S}$, we define the error probability as

$$
\begin{array}{r}
P_{k, m}(n)=\operatorname{Pr}\left[\rho \leftarrow \operatorname{Func}\left(\{0,1\}^{*},[m]^{k}\right) ; \operatorname{repr} \leftarrow \operatorname{ReP}^{\rho}(\mathcal{S}):\right. \\
\left.\operatorname{QRY}^{\rho}\left(\operatorname{repr}, \operatorname{qry}_{x}\right)>\operatorname{qry}_{x}(\mathcal{S})+\frac{e n}{m} \mid \operatorname{repr} \neq \perp\right] .
\end{array}
$$

(Here as above, $e$ denotes the base of the natural logarithm.) Informally, $P_{k, m}(n)$ is the probability that some $x$ is overestimated by a non-negligible amount in the representation of some $\mathcal{S}$ containing a total of $n$ elements, when a random function is used for hashing. Cormode and Muthukrishnan [8] show that this probability is bounded above by $e^{-k}$. This structure does not provide a bound for underestimation of frequencies, since it is designed for use cases where overestimates are considered harmful but underestimates are not.

ERROR FUNCTION FOR FREQUENCY QUERIES. The count min-sketch is designed for settings where overestimation in particular is undesirable, and so we aim to provide tight bounds on the size of overestimates but makes no guarantees about underestimates. To make the bounds simpler while staying conservative in our assumptions, we will use an error function that counts any overestimate as an error, not just overestimates larger than some lower bound of significance. In particular, we define $\delta$ as

$$
\delta(x, y)= \begin{cases}1 & \text { if } x>y \\ 0 & \text { otherwise }\end{cases}
$$

\subsection{Insecurity of public sketches}

Unlike in the Bloom filter case, good security bounds cannot be achieved for a count-min sketch in the ERR-Pub setting even if salts and/or private keys are used. The insecurity is due to the relative power of the Up oracle compared to the Bloom filter. Not only does it allow for deletion as well as insertion, but since updates are added to the representation rather than being combined with bitwise-OR, the adversary gains more information from seeing updates occur. Because of these differences, the adversary can mount an attack similar to the target-set coverage attack for a Bloom filter even if a PRF is used for hashing. First, the adversary calls $\operatorname{Rep}(\emptyset)$ to get an empty representation. The adversary can then call Up to insert an element into the set, see exactly what the outputs of each of the hash functions are, and then call $\mathbf{U p}$ again to delete the element. By doing this repeatedly, an adversary can determine the outputs of the PRF for $u$ different inputs using $2 u$ calls to $\mathbf{U p}$. Once a sufficiently large number of PRF outputs has been determined, the adversary can construct the test and target set used for the target-set coverage attack (Section 4.1). The adversary then calls Up several more times to insert each element of the test set into the sketch, and then each element of the target set will be overestimated.

In actual use, this specific attack may not be feasible for the adversary. However, as long as the sketch is public, the adversary can easily determine the exact results of inserting or deleting any element just by seeing which counters are incremented or decremented. For this reason it is not enough that the function used to perform queries and updates is impossible for the adversary to simulate, since the adversary can build a lookup table just by watching the sketch as it is updated. Instead, we must require that the sketch itself be kept secret from the adversary.

\subsection{Private, $\ell$-thresholded sketches}

Given the success of $\ell$-thresholding in the case of Bloom filters, we continue using this tweak in the case of count min-sketches. Between thresholding and the use of a per-representation random salt, we are able to establish an upper bound on the number of overestimates in a count min-sketch. However, the bound is not quite as good as in the case of a salted and thresholded Bloom filter, which is unsurprising given the increased flexibility provided by the update algorithm coupled with the additional information returned by the query evaluation algorithm. Formally, we consider the structure given by $\Pi=\operatorname{S\kappa ETch}[H, \ell, \lambda]$ for a hash function $H:\{0,1\}^{*} \rightarrow[m]^{k}$, which we will model as a random oracle.

Theorem 6.1 (ERR-Priv SECURITY OF THREsholded CMS). Let $p_{\ell}=((\ell+1) / m)^{k}$. For all $q_{R}, q_{T}, q_{U}, q_{H}, q_{V}, r, t \geq 0$ it holds that

$$
\begin{aligned}
\operatorname{Adv}_{\Pi, \delta, r}^{\text {err-priv }}\left(O(t), q_{R}, q_{T}, q_{U}, q_{H}, q_{V}\right) \leq \\
q_{R} \cdot\left[\frac{q_{H}}{2^{\lambda}}+e^{r^{\prime}-p_{\ell} q_{T}}\left(\frac{p_{\ell} q_{T}}{r^{\prime}}\right)^{r}\right],
\end{aligned}
$$

where $H$ is modeled as a random oracle, $r^{\prime}=\lfloor r /(k+1)\rfloor$, and $r^{\prime}>$ $p_{\ell} q_{T}$.

The theorem uses several reductions to gradually whittle away at the flexibility the adversary has in performing repeated insertions, deletions, and queries to the same elements. The $r^{\prime}$ in place of $r$ in the bound comes from the fact that, if the adversary finds that some $x$ is overestimated, it may be able to produce as many as $k$ additional overestimates by inserting $x$. We take this into account by automatically giving the adversary credit for all $k$ additional overestimates as soon as it discovers the false positive. After taking this into account, we can reduce to the standard binomial argument in which the adversary seeks to find $r^{\prime}$ overestimates by making arbitrary queries.

Proof Sketch of Theorem 6.1. Again we derive a bound in the ERR-Priv1 case and then use Lemma 4.2 to move from ERR-Priv1 to the more general ERR-Priv case. Because we are in the ERR-Priv1 case, we may assume without loss of generality that the adversary does not call Reveal, since revealing the only representation automatically prevents the adversary from winning.

As usual, we add a bad flag that gets set if the adversary ever guesses the salt when making a Hash query. By an almost identical argument, we can move to $\mathbf{G}_{1}$, where the behavior is different only 
when the bad flag is set, which occurs with probability no more than $q_{H} / 2^{\lambda}$.

The key differences between this proof and the Bloom filter proof are the more complex response space of Qry $(\mathbb{N}$ rather than $\{0,1\})$ and the fact that both elements of $\mathcal{S}$ and non-elements of $\mathcal{S}$ may produce errors.

In fact, deletion is never helpful to the adversary because deleting an element does not affect whether that element is overestimated and can only decrease the chance that other elements are overestimated. So we can assume that the adversary does not delete elements.

Using a similar argument, we can show that without loss of generality we may assume the adversary does not insert an element more than once. Since updates are deterministic, re-inserting $x$ can only increment the same counters that were incremented by the original insertion of $x$, and so this re-insertion cannot cause $y$ to become overestimated if it was not already.

As a third step, we move from $\mathbf{G}_{1}$ to a $\mathbf{G}_{2}$ where the adversary gains $k+1$ 'points' for finding a query which produces an overestimate, but which prevents the adversary from querying elements of $\mathcal{S}$. These extra points are necessary because, unlike in the case of a Bloom filter, inserting an overestimated element $x$ can cause other elements of $\mathcal{S}$ to become overestimated. In particular, if one of the counters incremented by the insertion of $x$ is shared with an element of $\mathcal{S}$ that is not overestimated, that element may become overestimated. However, if that counter is shared with multiple elements of $\mathcal{S}$, that counter is already an overestimate for all of the elements associated with it, and so no more than one overestimate can be caused per counter incremented by the insertion of $x$. Since inserting $x$ increments $k$ counters, at most $k$ errors can be caused in this way. Moving to this game allows us to assume that an adversary does not insert an element which is known to be overestimated, but does not decrease an adversary's chances of success.

We are now dealing with an adversary that gains points when it finds any overestimate, but which only makes queries to $x \notin \mathcal{S}$. This means that an error is simply a query that returns a value greater than 1. Analogously to the proof of Theorem 4.5, we now move to a game $\mathbf{G}_{3}$ where $\mathbf{R e p}$ randomly fills the sketch to capacity after inserting the elements of $\mathcal{S}$, so that each row has $\ell+1$ non-zero counters. Again this does not reduce the adversary's chances of success, but allows us to assume that the adversary never calls Up

The probability of the adversary winning can now be given by another binomial bound. The set of nonzero counters in each row is a uniformly random subset of $[m]$ of size $\ell+1$. Since any query returning a nonzero value is a success for the adversary, the probability of any particular Qry call causing a collision within a single row $i$ is $(\ell+1) / m$, and the probability of a collision in every row (i.e. an error) is $((\ell+1) / m)^{k}$. The adversary has a total of $q_{T}$ attempts, and wins if it accumulates $\lfloor r /(k+1)\rfloor$ successes, since each error gives it $k+1$ points. So, letting $p_{\ell}=((\ell+1) / m)^{k}$ and $r^{\prime}=\lfloor r /(k+1)\rfloor$, we apply the usual Chernoff bound and Lemma 4.2 to derive the final bound of

$$
\operatorname{Adv}_{\Pi, \delta, r}^{\text {err-priv }}(A) \leq q_{R} \cdot\left[\frac{q_{H}}{2^{\lambda}}+e^{r^{\prime}-p_{\ell} q_{T}}\left(\frac{p_{\ell} q_{T}}{r^{\prime}}\right)^{r}\right] .
$$

\subsection{Discussion}

Unlike Bloom filters, there is no simple tweak that can be performed to a count min-sketch to provide good ERR-Pub security bounds. In particular, it does not achieve security even in the immutable setting, and adding a secret key does not help. However, the bound above shows that in settings where sketches can be assumed secret it is possible to prove an upper bound on the number of overestimates an adversary can cause. In particular, we recommend the combination of random per-representation salts and $\ell$-thresholding in order to mitigate possible attacks in the ERR-Priv setting.

The bound we achieve is based on the same binomial bound as in the case of Bloom filters, but has a notable difference in the form of $r^{\prime}$ replacing $r$. This negatively impacts the amount of space the filter must take up in order to provide low error bounds, but because the scaling factor between $r$ and $r^{\prime}$ is only $k+1$, the difference should not be unacceptably extreme given reasonable parameter choices. We also note that it is possible this bound can be improved to reduce the impact on sketch size, since the initial factor of $q_{R}$ does not have an obvious attack associated with it which would make this bound tight. (The same is true, of course, of Bloom filters.)

\section{REFERENCES}

[1] Mihir Bellare and Phillip Rogaway. 1993. Random Oracles Are Practical: A Paradigm for Designing Efficient Protocols. In Proceedings of the 1st ACM Conference on Computer and Communications Security (CCS '93). ACM, New York, NY, USA, 62-73. https://doi.org/10.1145/168588.168596

[2] Mihir Bellare and Phillip Rogaway. 2006. The Security of Triple Encryption and a Framework for Code-based Game-playing Proofs. In EUROCRYPT 2006: Proceedings of the 24th Annual International Conference on The Theory and Applications of Cryptographic Techniques.

[3] Steven M. Bellovin and William R. Cheswick. 2004. Privacy-Enhanced Searches Using Encrypted Bloom Filters. Cryptology ePrint Archive, Report 2004/022. http://eprint.iacr.org/2004/022.

[4] Burton H. Bloom. 1970. Space/time trade-offs in hash coding with allowable errors. Commun. ACM 13, 7 (1970).

[5] Andrei Broder and Michael Mitzenmacher. 2004. Network Applications of Bloom Filters: A Survey. Internet Mathematics 1, 4 (2004)

[6] John W. Byers, Jeffrey Considine, Michael Mitzenmacher, and Stanislav Rost. 2004. Informed Content Delivery Across Adaptive Overlay Networks. IEEE/ACM Trans. Netw. 12, 5 (2004).

[7] Bernard Chazelle, Joe Kilian, Ronitt Rubinfeld, and Ayellet Tal. 2004. The Bloomier Filter: An Efficient Data Structure for Static Support Lookup Tables. In SODA 2004: Proceedings of the 15th Annual ACM-SIAM Symposium on Discrete Algorithms.

[8] Graham Cormode and S Muthukrishnan. 2005. An improved data stream summary: The count-min sketch and its applications. Fournal of Algorithms 55, 1 (2005)

[9] Scott A. Crosby and Dan S. Wallach. 2003. Denial of Service via Algorithmic Complexity Attacks. In Proceedings of the 12th USENIX Security Symposium.

[10] Jeffrey Dean and Sanjay Ghemawat. 2008. MapReduce: simplified data processing on large clusters. Commun. ACM 51, 1 (2008).

[11] Fan Deng and Davood Rafiei. 2006. Approximately detecting duplicates for streaming data using stable bloom filters. In Proceedings of the 2006 ACM SIGMOD international conference on Management of data. ACM, 25-36.

[12] Martin Dietzfelbinger and Rasmus Pagh. 2008. Succinct Data Structures for Retrieval and Approximate Membership (Extended Abstract). In ICALP 2008: Proceedings of the 35th International Colloquium on Automata, Languages and Programming.

[13] Marianne Durand and Philippe Flajolet. 2003. Loglog Counting of Large Cardinalities. In ESA 2003: Proceedings of the 11th Annual European Symposium on Algorithms.

[14] Bin Fan, David G. Andersen, Michael Kaminsky, and Michael D. Mitzenmacher. 2014. Cuckoo filter: Practically better than bloom. In Proceedings of the 10th ACM International Conference on Emerging Networking Experiments and Technologies.

[15] Li Fan, Pei Cao, Jussara Almeida, and Andrei Z Broder. 2000. Summary cache: A scalable wide-area web cache sharing protocol. IEEE/ACM Transactions on Networking 8, 3 (2000).

[16] Wu-chang Feng, Dilip D. Kandlur, Debanjan Saha, and Kang G. Shin. 2001. Stochastic Fair Blue: A Queue Management Algorithm for Enforcing Fairness. In 
INFOCOM 2001: Proceedings of the 20th Annual foint Conference of the IEEE Computer and Communications Society.

[17] Michael L. Fredman, János Komlós, and Endre Szemerédi. 1984. Storing a Sparse Table with 0(1) Worst Case Access Time. 7. ACM 31, 3 (1984).

[18] Thomas Gerbet, Amrit Kumar, and Cédric Lauradoux. 2015. The Power of Evil Choices in Bloom Filters. In Proceedings of the 45th Annual IEEE/IFIP International Conference on Dependable Systems and Networks.

[19] Arthur Gervais, Srdjan Capkun, Ghassan O Karame, and Damian Gruber. 2014. On the privacy provisions of Bloom filters in lightweight Bitcoin clients. In ACSAC 2014: Proceedings of the 30th Annual Computer Security Applications Conference.

[20] Adam Kirsch and Michael Mitzenmacher. 2008. Less Hashing, Same Performance: Building a Better Bloom Filter. Random Structures and Algorithms 33, 2 (2008).

[21] James Larisch, David Choffnes, Dave Levin, Bruce M. Maggs, Alan Mislove, and Christo Wilson. 2017. CRLite: A Scalable System for Pushing All TLS Revocations to All Browsers. In The Proceedings of the 38th IEEE Symposium on Security and Privacy.

[22] Richard J. Lipton and Jeffrey F. Naughton. 1993. Clocked Adversaries for Hashing Algorithmica 9, 3 (1993).

[23] Ilya Mironov, Moni Naor, and Gil Segev. 2011. Sketching in Adversarial Environments. SIAM 7. Comput. 40, 6 (2011).

[24] Moni Naor and Eylon Yogev. 2015. Bloom Filters in Adversarial Environments. In CRYPTO 2015: Proceedings of the 35th Annual Cryptology Conference.

[25] Ryo Nojima and Youki Kadobayashi. 2009. Cryptographically Secure BloomFilters. Transactions on Data Privacy 2, 2 (2009).

[26] Patrick Reynolds and Amin Vahdat. 2003. Efficient peer-to-peer keyword searching. In Proceedings of the ACM/IFIP/USENIX 2003 International Conference on Middleware.

[27] Rainer Schnell, Tobias Bachteler, and Jörg Reiher. 2011. A novel error-tolerant anonymous linking code. Working paper series no. WP-GRLC-2011-02, German Record Linkage Center.

[28] Sasu Tarkoma, Christian Rothenberg, and Eemil Lagerspetz. 2012. Theory and Practice of Bloom Filters for Distributed Systems. IEEE Communications Surveys and Tutorials 14, 1 (2012).

\section{A A GENERIC ERR-PRIV1 ATTACK AGAINST BASIC BLOOM FILTERS}

Fix integers $\ell, n, \lambda \geq 0$, let $H:\{0,1\}^{*} \rightarrow[m]^{k}$ be a function, and let $\Pi=\mathrm{BF}[H, t, \lambda]$ as specified in Figure 5 . The possibility of precomputing the structure in the ERR-Pub1 games yields the following attack. Given a set $\mathcal{T} \subseteq\{0,1\}^{*}$ of target queries, the adversary searches for a set $\mathcal{S} \subseteq\{0,1\}^{*}$ such that $\mathrm{QRY}^{H}\left(\operatorname{ReP}^{H}(\mathcal{S}), x\right)=1$ for all $x \in \mathcal{T}$. We call such a set a cover set. Once a suitable $\mathcal{S}$ is found, the adversary queries $\operatorname{Rep}(\mathcal{S})$ followed by $\operatorname{Qry}(x)$ for each $x \in \mathcal{T}$. Assuming $|\mathcal{T}| \geq r$, where $r$ is the error capacity, the attack succeeds with probability 1 . In this appendix we describe a strategy for finding a covering set and evaluate its performance in terms of success rate and computational cost. We will (conservatively) model $H$ as a random oracle.

We first choose a set $\mathcal{S}$ of $s>n$ potential test queries. Our goal is to find a subset of $\mathcal{S}$ that covers $\mathcal{T}$ but contains at most $n$ elements. For each test query $x$, we compute $X=B_{m}(\operatorname{Hash}(x))$. If we have the resources to compute $X_{1} \vee \cdots \vee X_{n}$ for each of the $\left(\begin{array}{l}s \\ n\end{array}\right)$ size- $n$ subsets of targets, we will eventually find a suitable covering set if such a set exists. The query complexity of this approach is modest; we need to make $s+r$ queries to Hash ( $s$ for the test set, $r$ for the target set), one query to Rep, and $r$ queries to Qry. However, checking $\left(\begin{array}{l}s \\ n\end{array}\right)$ sets would be infeasible, even for modest choices of $k, m$, and $n$. Observe, however, that there is a lot of sub-structure to exploit in the search. In the remainder, we describe a heuristic strategy for finding a satisfying set (if it exists) in which we need only check about $O(k r)$ sets on average.

Let $\mathcal{S}=\left\{x_{1}, \ldots, x_{s}\right\}$ be the set of potential test queries, and let $\mathcal{T}=\left\{x_{s+1}, \ldots, x_{s+r}\right\}$ be the set of target queries. We construct a tree whose vertices are labeled with subsets of $[s]$ as follows. Let $\emptyset$ be the root. For each node $I$ and each $w \in[s] \backslash I$, if $|I|<n$, then let
$I \cup\{w\}$ be a child of $I$. The new attack works as follows: traverse the tree depth-first, beginning at $\emptyset$, until a vertex $I$ is reached such that each element of $\mathcal{T}$ is a false positive for the representation of $\mathcal{S}_{I}=\left\{x_{i}: i \in I\right\} \subseteq \mathcal{S}$. Then for every child $J$ of $I$, the elements of $\mathcal{T}$ are false positives for $\mathcal{S}_{J}$. The adversary may choose any one of these as its query to Rep.

The tree has $\left(\begin{array}{l}s \\ n\end{array}\right)$ leaves, and it will traverse the entire tree if there is no solution. Hence, the worst-case runtime is the same as before. However, we can reduce the search space dramatically in two ways. First, if there is no solution, then often this can be determined without traversing the tree. Let $M_{\mathcal{S}}$ and $M_{\mathcal{T}}$ denote the filters corresponding to the set of test and target queries, respectively. If for some $i \in[m]$, the $i$-th bit of $M_{\mathcal{T}}$ is set, but the $i$-th bit of $M_{\mathcal{S}}$ is not set, then it is immediate that there is no covering set. The second is a greedy heuristic for eliminating branches of the search tree. The idea is that we only take a branch if it results in covering an additional bit in $M_{\mathcal{T}}$. More precisely, for each child $J$ of $I$, we do as follows: if there is a bit $i$ such that the $i$-th bit of $M_{\mathcal{S}_{J}}$ and $M_{\mathcal{T}}$ are set, but the $i$-th bit of $M_{\mathcal{S}_{I}}$ is not set, then the branch $J$ is taken; otherwise it is not. This optimization results in a heuristic attack, since the search may miss the optimal solution.

We implemented this attack and evaluated its performance. Figure 11 shows the success rate and average number of sets checked for a number of simulations and various parameters. Unsurprisingly, the success rate decreases as we increase the number of filter bits (top-left of Figure 11); however, with $k=4, m=1024, n=100$, and $r=1$, a test set of just 512 elements suffices for a success rate of nearly $60 \%$ (top-right). It is also worth noting that increasing the error parameter only slightly decreases the success rate (bottomleft). These results show that even for very pessimistic parameter choices, the basic Bloom filter is not secure in the ERR-Pub1 sense. Finally, we find that the average number of sets that were tested is about $O(k r)$ within all of the parameter ranges we tested. 
Figure 11: Success rate and average-case time complexity for the optimized attack on classic Bloom filter. Each plot shows the success rate $(\bullet)$, as well as the average number of tested sets $(x)$, for 1000 executions of the attack on simulated inputs. Default parameters are $k=4, m=2^{10}$, $n=100, r=1$, and $s=2^{10}$. In each plot, one of these parameters is varied.
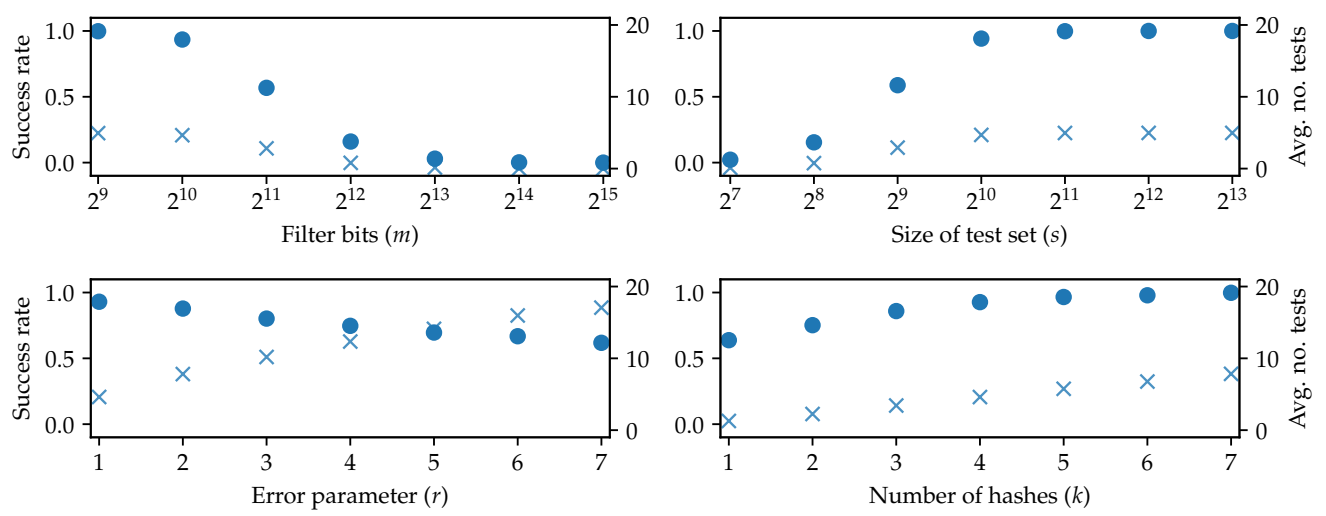